\title{
A Search for Fast Optical Transients in the Pan- STARRS1 Medium-Deep Survey: M-Dwarf Flares, Asteroids, Limits on Extragalactic Rates, and Implications for LSST
}

\section{Citation}

Berger, E., C. N. Leibler, R. Chornock, A. Rest, R. J. Foley, A. M. Soderberg, P. A. Price, et al. 2013. "A Search for Fast Optical Transients in the Pan-STARRS1 Medium-Deep Survey: M-Dwarf Flares, Asteroids, Limits on Extragalactic Rates, and Implications for LSST." The Astrophysical Journal 779 (1) (December 10): 18. doi:10.1088/0004-637x/779/1/18. http:// dx.doi.org/10.1088/0004-637X/779/1/18.

\section{Published Version}

doi:10.1088/0004-637x/779/1/18

\section{Permanent link}

http://nrs.harvard.edu/urn-3:HUL.InstRepos:11829464

\section{Terms of Use}

This article was downloaded from Harvard University's DASH repository, and is made available under the terms and conditions applicable to Other Posted Material, as set forth at http:// nrs.harvard.edu/urn-3:HUL.InstRepos:dash.current.terms-of-use\#LAA

\section{Share Your Story}

The Harvard community has made this article openly available.

Please share how this access benefits you. Submit a story.

\section{Accessibility}




\title{
A SEARCH FOR FAST OPTICAL TRANSIENTS IN THE Pan-STARRS1 MEDIUM-DEEP SURVEY: M-DWARF FLARES, ASTEROIDS, LIMITS ON EXTRAGALACTIC RATES, AND IMPLICATIONS FOR LSST
}

\author{
E. Berger ${ }^{1}$, C. N. Leibler ${ }^{1,2}$, R. Chornock ${ }^{1}$, A. Rest $^{3}$, R. J. Foley ${ }^{1}$, A. M. Soderberg ${ }^{1}$, P. A. Price ${ }^{4}$, W. S. BurgetT ${ }^{5}$, \\ K. C. Chambers ${ }^{5}$, H. Flewelling ${ }^{5}$, M. E. Huber ${ }^{5}$, E. A. Magnier ${ }^{5}$, N. Metcalfe $^{6}$, C. W. Stubis ${ }^{7}$, and J. L. Tonry ${ }^{5}$ \\ ${ }^{1}$ Harvard-Smithsonian Center for Astrophysics, 60 Garden Street, Cambridge, MA 02138, USA \\ ${ }^{2}$ Department of Astronomy and Astrophysics, University of California, Santa Cruz, CA 95064, USA \\ ${ }^{3}$ Space Telescope Science Institute, 3700 San Martin Drive, Baltimore, MD 21218, USA \\ ${ }^{4}$ Department of Astrophysical Sciences, Princeton University, Princeton, NJ 08544, USA \\ ${ }^{5}$ Institute for Astronomy, University of Hawaii, 2680 Woodlawn Drive, Honolulu, HI 96822, USA \\ ${ }^{6}$ Department of Physics, Durham University, South Road, Durham DH1 3LE, UK \\ ${ }^{7}$ Department of Physics, Harvard University, Cambridge, MA 02138, USA \\ Received 2013 July 19; accepted 2013 October 1; published 2013 November 19
}

\begin{abstract}
We present a search for fast optical transients ( $\tau \sim 0.5 \mathrm{hr}-1$ day) using repeated observations of the Pan-STARRS1 Medium-Deep Survey (PS1/MDS) fields. Our search takes advantage of the consecutive $g_{\mathrm{P} 1} r_{\mathrm{P} 1}$ observations (16.5 minutes in each filter), by requiring detections in both bands, with non-detections on preceding and subsequent nights. We identify 19 transients brighter than $22.5 \mathrm{AB}$ mag $(\mathrm{S} / \mathrm{N} \gtrsim 10)$. Of these, 11 events exhibit quiescent counterparts in the deep PS1/MDS templates that we identify as M4-M9 dwarfs at $d \approx 0.2-1.2 \mathrm{kpc}$. The remaining eight transients lack quiescent counterparts, exhibit mild but significant astrometric shifts between the $g_{\mathrm{P} 1}$ and $r_{\mathrm{P} 1}$ images, colors of $(g-r)_{\mathrm{P} 1} \approx 0.5-0.8 \mathrm{mag}$, non-varying light curves, and locations near the ecliptic plane with solar elongations of about $130^{\circ}$, which are all indicative of main-belt asteroids near the stationary point of their orbits. With identifications for all 19 transients, we place an upper limit of $R_{\text {FOT }}(\tau \sim 0.5 \mathrm{hr}) \lesssim 0.12 \mathrm{deg}^{-2} \mathrm{day}^{-1}$ (95\% confidence level) on the sky-projected rate of extragalactic fast transients at $\lesssim 22.5$ mag, a factor of 30-50 times lower than previous limits; the limit for a timescale of $\sim 1$ day is $R_{\mathrm{FOT}} \lesssim 2.4 \times 10^{-3} \mathrm{deg}^{-2} \mathrm{day}^{-1}$. To convert these sky-projected rates to volumetric rates, we explore the expected peak luminosities of fast optical transients powered by various mechanisms, and find that non-relativistic events are limited to $M \approx-10$ to $\approx-14$ mag for a timescale of $\sim 0.5 \mathrm{hr}$ to $\sim 1$ day, while relativistic sources (e.g., gamma-ray bursts, magnetar-powered transients) can reach much larger luminosities. The resulting volumetric rates are $\lesssim 13 \mathrm{Mpc}^{-3} \mathrm{yr}^{-1}(M \approx-10 \mathrm{mag})$, $\lesssim 0.05 \mathrm{Mpc}^{-3} \mathrm{yr}^{-1}(M \approx-14 \mathrm{mag})$, and $\lesssim 10^{-6} \mathrm{Mpc}^{-3} \mathrm{yr}^{-1}(M \approx-24 \mathrm{mag})$, significantly above the nova, supernova, and gamma-ray burst rates, respectively, indicating that much larger surveys are required to provide meaningful constraints. Motivated by the results of our search, we discuss strategies for identifying fast optical transients in the Large Synoptic Survey Telescope main survey, and reach the optimistic conclusion that the veil of foreground contaminants can be lifted with the survey data, without the need for expensive follow-up observations.
\end{abstract}

Key words: minor planets, asteroids: general - novae, cataclysmic variables - stars: flare - supernovae: general surveys

Online-only material: color figures

\section{INTRODUCTION}

For nearly a century, optical observations aimed at the discovery and study of astrophysical transients have largely focused on events with durations of days to months. This is mainly due to a fortuitous match with the timescales of the most common extragalactic events (novae and supernovae), whose typical luminosities and intrinsic rates require coverage of large numbers of galaxies and/or blank sky areas, leading to a natural search cadence of several days. Thus, novae have a much higher intrinsic rate than supernovae $\left(\sim 2.2 \mathrm{yr}^{-1}\right.$ per $10^{10} L_{K, \odot}$ versus $\sim 2 \times 10^{-3} \mathrm{yr}^{-1}$ per $10^{10} L_{K, \odot}$, respectively; e.g., Williams \& Shafter 2004; Li et al. 2011), but supernovae are significantly more luminous than novae $(\sim-18$ mag versus $\sim-8$ mag, respectively; Gallagher \& Starrfield 1978; Filippenko 1997). As a result, for a given survey limiting magnitude, tens of novae can be discovered per year by targeting a few nearby galaxies with a cadence of few days, while discovering a similar number of supernovae requires monitoring of $\sim 10^{4}$ galaxies (or hundreds of $\mathrm{deg}^{2}$ ), thereby necessitating a similar cadence of several days; for the purpose of sheer discovery rate, a faster cadence is not profitable for nova and supernova searches. Over the past few decades, such surveys have been highly successful at discovering about 100 novae and supernovae per year (e.g., Williams \& Shafter 2004; Leaman et al. 2011).

The advent of large format cameras on dedicated wide-field telescopes, coupled with serendipitous discoveries of transients outside of the traditional nova and supernova luminosity and timescale ranges, has opened up a new discovery space for astrophysical transients. By repeatedly targeting the same fields, such surveys are in principle capable of exploring a wide range of timescales, from the duration of single exposures (i.e., minutes) to years. In practice, most surveys are still primarily focused on supernovae (driven to a large extent by Type Ia supernova cosmology at increasingly larger redshifts), and therefore cover wider fields to greater depth at the expense of a faster temporal cadence to maximize the supernova discovery rate while preserving adequate light-curve coverage. Still, some surveys have been utilized to perform initial searches for fast optical transients (FOTs) on timescales as short as $\sim 0.5 \mathrm{hr}$. Clearly, the effective areal exposure of such searches (i.e., the product of survey area and exposure time) becomes progressively smaller 
at faster cadence as sky coverage has to be sacrificed for repeated short-cadence observations.

In this context, the Deep Lens Survey (DLS) was utilized to search for FOTs on a timescale of about $1300 \mathrm{~s}$ to a depth of $B \approx 23.8 \mathrm{mag}$ (with a total exposure of $1.1 \mathrm{deg}^{2}$ day) and led to an upper limit on the extragalactic sky-projected rate of $R_{\mathrm{FOT}} \lesssim 6.5 \mathrm{deg}^{-2} \mathrm{day}^{-1}$ (95\% confidence level; Becker et al. 2004). The DLS search uncovered three fast transients, which were shown to be flares from Galactic M-dwarf stars (Becker et al. 2004; Kulkarni \& Rau 2006). A search for transients with a timescale of $\gtrsim 0.5 \mathrm{hr}$ to a depth of about $17.5 \mathrm{mag}$ (with a total exposure of $635 \mathrm{deg}^{2}$ day) using the Robotic Optical Transient Search Experiment-III (ROTSE-III) yielded a limit on the extragalactic rate of $R_{\mathrm{FOT}} \lesssim 5 \times 10^{-3} \mathrm{deg}^{-2} \mathrm{day}^{-1}$, and uncovered a single M-dwarf flare (Rykoff et al. 2005); a similar search with the MASTER telescope system yielded comparable limits (Lipunov et al. 2007), and two uncharacterized candidate fast transients (Gorbovskoy et al. 2013). Similarly, a targeted search for transients on a timescale of about $0.5 \mathrm{hr}$ and to a depth of $B \approx 21.3$ mag in the Fornax galaxy cluster (with a total exposure of $1.9 \mathrm{deg}^{2}$ day) placed a limit on the extragalactic rate of $R_{\text {FOT }} \lesssim 3.3 \mathrm{deg}^{-2}$ day $^{-1}$ (Rau et al. 2008). Two fast transients were detected in this search, both shown to be M-dwarf flares (Rau et al. 2008). At the bright end, the "Pi of the Sky" project placed a limit on transients brighter than $11 \mathrm{mag}$ with a duration of $\gtrsim 10$ s of $R_{\text {FOT }} \lesssim 5 \times 10^{-5} \mathrm{deg}^{-2}$ day $^{-1}$ (Sokołowski et al. 2010).

FOTs have also been found serendipitously by other surveys, but they have generally been shown to be Galactic in origin. ${ }^{8}$ A notable exception is the transient PTF11agg (Cenko et al. 2013), which faded by about $1.2 \mathrm{mag}$ in $5.3 \mathrm{hr}$, and $3.9 \mathrm{mag}$ in 2.2 days, and was accompanied by radio emission that may be indicative of relativistic expansion (although the distance of this transient is not known, thereby complicating its interpretation). We note that such an event, while fast compared to the general nova and supernova population, is still of longer duration than the timescales probed by the DLS and Fornax searches, as well as the Pan-STARRS1 search we describe here. Thus, the existing searches for extragalactic FOTs have mainly raised the awareness that the foreground of M-dwarf flares is large, with an estimated all-sky rate of $\sim 10^{8} \mathrm{yr}^{-1}$ at a limiting magnitude of 24 mag (Becker et al. 2004; Kulkarni \& Rau 2006).

Here, we present a search for FOTs with an effective timescale of about $0.5 \mathrm{hr}$ to 1 day and to a depth of about $22.5 \mathrm{mag}$ in the first $1.5 \mathrm{yr}$ of data from the Pan-STARRS1 Medium-Deep Survey (PS1/MDS). This search uncovered a substantial sample of 19 fast transients, both with and without quiescent counterparts. We describe the survey strategy and selection criteria in Section 2. In Section 3, we summarize the properties of the 19 detected transients and classify them using a combination of color information, astrometry, sky location, and the properties of quiescent counterparts (when detected). With a unique identification of all 19 transients as solar system or Galactic in origin, we place a limit on the rate of extragalactic fast transients that is 30-50 times better than the limits from previous searches (Section 4). We further investigate for the first time the limits on the volumetric rates of FOTs from our survey and previous searches using the survey limiting magnitudes and fiducial tran-

\footnotetext{
8 A recent example is a large amplitude transient from the Catalina Real-Time Transient Survey, which was initially claimed to be extragalactic in origin (Mahabal et al. 2012), but was subsequently shown to be an M-dwarf flare (Berger et al. 2012). Additional cautionary tales are summarized by Kulkarni \& Rau (2006).
}

sients luminosities. In Section 5, we expand on this point and discuss the expected peak luminosities of FOTs for a range of physically motivated models. Finally, since our search is the first one to utilize observations that are similar to the Large Synoptic Survey Telescope (LSST) main survey strategy, we conclude by drawing implications for FOT searches in the LSST data in Section 6.

\section{OBSERVATIONS}

\subsection{PS1 Survey Summary}

The PS1 telescope, located on Mount Haleakala, is a high-etendue wide-field survey instrument with a $1.8-\mathrm{m} \mathrm{di}$ ameter primary mirror and a 3.3 diameter field of view imaged by an array of sixty $4800 \times 4800$ pixel detectors, with a pixel scale of 0'.258 (Kaiser et al. 2010; Tonry \& Onaka 2009). The observations are obtained through five broad-band filters $\left(g_{\mathrm{P} 1} r_{\mathrm{P} 1} i_{\mathrm{P} 1} z_{\mathrm{P} 1} y_{\mathrm{P} 1}\right)$, with some differences relative to the Sloan Digital Sky Survey (SDSS); the $g_{\mathrm{P} 1}$ filter extends $200 \AA$ redward of $g_{\text {SDSS }}$ to achieve greater sensitivity and lower systematics for photometric redshifts, while the $z_{\mathrm{P} 1}$ filter terminates at $9300 \AA$, unlike $z_{\text {SDSS }}$ which is defined by the detector response (Tonry et al. 2012). PS1 photometry is in the "natural" system, $m=-2.5 \log \left(F_{v}\right)+m^{\prime}$, with a single zero-point adjustment $\left(m^{\prime}\right)$ in each band to conform to the AB magnitude scale. Magnitudes are interpreted as being at the top of the atmosphere, with 1.2 airmasses of atmospheric attenuation included in the system response function (Tonry et al. 2012).

The PS1/MDS consists of 10 fields (each with a single PS1 imager footprint) observed on a nearly nightly basis by cycling through the five filters in three to four nights to a typical $5 \sigma$ depth of $\sim 23.3 \mathrm{mag}$ in $g_{\mathrm{P} 1} r_{\mathrm{P} 1} i_{\mathrm{P} 1} z_{\mathrm{P} 1}$, and $\sim 21.7 \mathrm{mag}$ in $y_{\mathrm{P} 1}$. The MDS images are processed through the Image Processing Pipeline (IPP; Magnier 2006), which includes flat-fielding ("detrending"), a flux-conserving warping to a sky-based image plane, masking and artifact removal, and object detection and photometry. For the fast transient search described here we produced difference images from the stacked nightly images using the photpipe pipeline (Rest et al. 2005) running on the Odyssey computer cluster at Harvard University.

\subsection{A Search for Fast Optical Transients}

For the purpose of detecting FOTs, we take advantage of the consecutive MDS $g_{\mathrm{P} 1} r_{\mathrm{P} 1}$ observations, with eight $113 \mathrm{~s}$ exposures in each filter providing a total time-span of about 33 minutes for a full sequence. We carry out the search using the stacked $g_{\mathrm{P} 1}$ and $r_{\mathrm{P} 1}$ images from each visit through image subtraction relative to deep multi-epoch templates, and subsequently utilize the individual exposures to construct light curves; representative discovery and template images are shown in Figure 1 and light curves are shown in Figure 2. We limit the timescale of the transients to $\lesssim 1$ day by further requiring no additional detections in the $g_{\mathrm{P} 1} r_{\mathrm{P} 1} i_{\mathrm{P} 1} z_{\mathrm{P} 1}$ filters on preceding and subsequent nights (extending to \pm 5 nights). To ensure that this constraint is met, we only perform our search on the subset of MDS data for which consecutive nights of observations are available. In the first $1.5 \mathrm{yr}$ of data, we searched a total of 277 nights of $g_{\mathrm{P} 1} r_{\mathrm{P} 1}$ observations across the $10 \mathrm{MDS}$ fields, leading to a total areal exposure of $40.4 \mathrm{deg}^{2}$ day for a timescale of $0.5 \mathrm{hr}$ and $1940 \mathrm{deg}^{2}$ day for a timescale of 1 day; here, the areal exposure is simply the product of the total number of nights, the field of view per field, and the duration of the transient. 

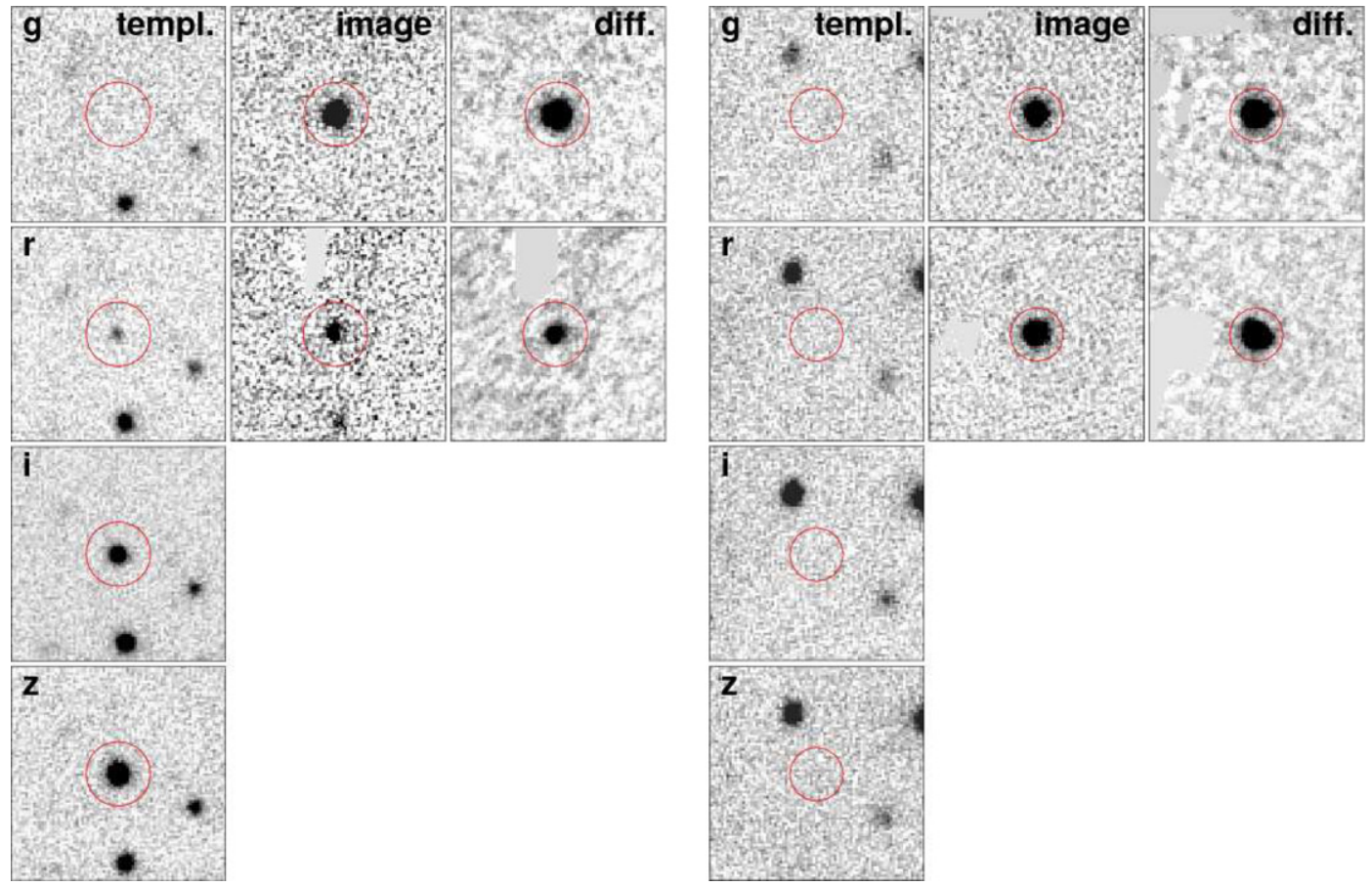

Figure 1. PS1/MDS images of a representative M-dwarf flare (left; PSO J164.3814+58.3011) and an asteroid (right; PSO J352.5968-0.4471) found in our fast transients search. In each case, we show the template images $\left(g_{\mathrm{P} 1} r_{\mathrm{P} 1} i_{\mathrm{P} 1} z_{\mathrm{P} 1}\right)$ in the left column, the fast transient discovery images in the middle column, and the difference images in the right column. Each panel is $20^{\prime \prime} \times 20^{\prime \prime}$ oriented with north up and east to the left. The red optical colors of the M-dwarf counterpart are evident, as is the lack of a counterpart in the case of the asteroid. The light curves resulting from these detections are shown in Figure 2.

(A color version of this figure is available in the online journal.)

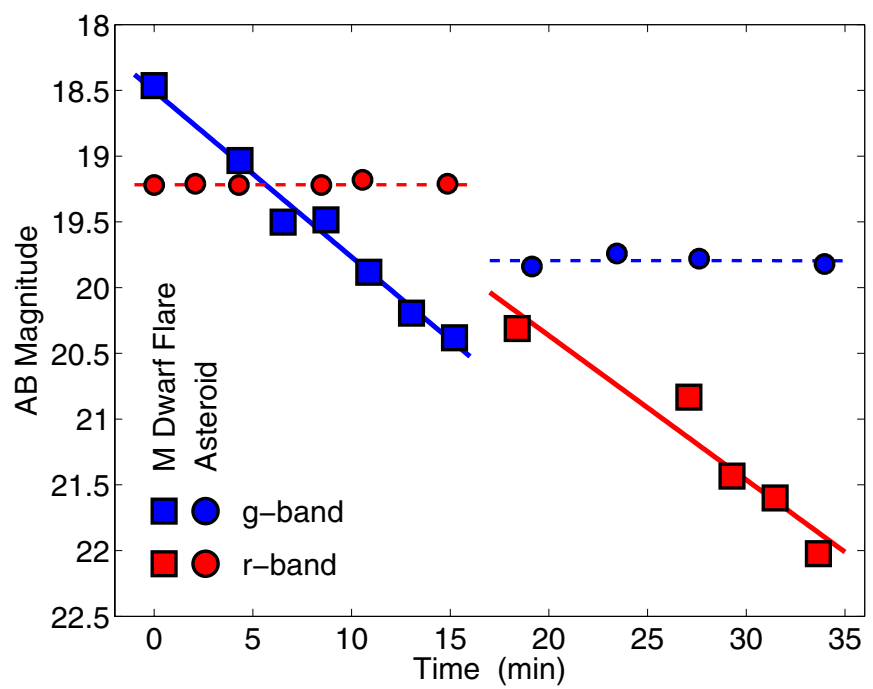

Figure 2. Light curves in the $g_{\mathrm{P} 1}$ (blue) and $r_{\mathrm{P} 1}$ (red) filters for a representative M-dwarf flare (squares) and an asteroid (circles) detected in our search for fast optical transients (see Figure 1). In each case, the lines mark the best linear fit. The M-dwarf flare exhibits a $3.5 \mathrm{mag}$ decline during the time-span of our observation, while the asteroid exhibits constant brightness. Missing light-curve points are due to individual exposures in which the source was located in a chip gap.

(A color version of this figure is available in the online journal.)

To select transients in the $g_{\mathrm{P} 1} r_{\mathrm{P} 1}$ difference images, we utilize a signal-to-noise ratio $(\mathrm{S} / \mathrm{N})$ threshold $^{9}$ of $\mathrm{S} / \mathrm{N}=10$,

\footnotetext{
9 We empirically correct the correlated noise in the difference image detections by measuring the flux and uncertainty at random positions in the difference images in the same manner as the transient flux, and then determining a correction factor, which leads to a distribution with a reduced $\chi^{2}$ of unity.
}

with resulting limiting magnitudes of $g_{\mathrm{P} 1} \approx 22.7 \mathrm{mag}$ and $r_{\mathrm{P} 1} \approx 22.4 \mathrm{mag}$. We additionally require sources in the $g_{\mathrm{P} 1} r_{\mathrm{P} 1}$ difference images to astrometrically match within 0.35 , corresponding to a 1.2 pixel radius around each detection (this is about six times the typical astrometric error; see Section 3.1). Using these cuts, we find a total of 227 candidates, which were visually inspected by one of us (C.L.) leading to a final list of 19 sources that were further validated by two of us (E.B. and R.C.); the remaining 208 events were predominantly spurious detections near saturated stars.

\section{THE PROPERTIES OF Pan-STARRS1 FAST OPTICAL TRANSIENTS}

Using the procedure described in the previous section, we found 19 genuine fast transients, spanning a brightness range of $g_{\mathrm{P} 1} \approx 18.5-22.7 \mathrm{mag}$ and $r_{\mathrm{P} 1} \approx 18.8-22.4 \mathrm{mag}$. In Figure 3 , we show the $g_{\mathrm{P} 1}$ versus $(g-r)_{\mathrm{P} 1}$ color-magnitude diagram for all 19 sources; the photometry is summarized in Tables 1 and 2. The faint end of the distribution is determined by our requirement of $S / N \gtrsim 10$ in the subtractions of the individual $g_{\mathrm{P} 1} r_{\mathrm{P} 1}$ nightly stacks (16.5 minutes in each filter) from the deep templates. On the other hand, the bright end of the observed distribution is about 2 mag fainter than the saturation limit of our images, indicating a genuine dearth of apparently bright fast transients in our search area. The $(g-r)_{\mathrm{P} 1}$ colors span a wide range of about -2.0 to $+0.9 \mathrm{mag}$, but we stress that for transients that rapidly vary in brightness within the time-span of each observation (e.g., Figure 2), the non-simultaneous $g_{\mathrm{P} 1}$ and $r_{\mathrm{P} 1}$ measurements do not reflect the true instantaneous colors.

\subsection{Fast Transients Lacking Quiescent Counterparts}

Of the 19 fast transients discovered in our search, 8 events lack quiescent counterparts in any of the deep template images 
Table 1

Fast Optical Transients with Quiescent Counterparts

\begin{tabular}{|c|c|c|c|c|c|c|c|c|}
\hline OBJID & $\begin{array}{l}\text { R.A. } \\
(\mathrm{h} \mathrm{m} \mathrm{s})\end{array}$ & $\begin{array}{l}\text { Decl. } \\
\left(0^{\prime \prime \prime}\right)\end{array}$ & UT Date & $\begin{array}{c}m_{\text {flare,P1 }} \\
\text { (AB mag) }\end{array}$ & $\begin{array}{c}m_{\mathrm{q}, \mathrm{P} 1} \\
(\mathrm{AB} \mathrm{mag})\end{array}$ & $\begin{array}{c}m_{\mathrm{q}, \mathrm{SDSS}} \\
(\mathrm{AB} \mathrm{mag})\end{array}$ & Sp. T. & $\begin{array}{c}d \\
(\mathrm{pc})\end{array}$ \\
\hline PSO J52.0080-27.0515 & $03^{\mathrm{h}} 28^{\mathrm{m}} 01^{\mathrm{s}} .929$ & $-27^{\circ} 03^{\prime} 05^{\prime \prime} .51$ & 2010 Sep 7 & $\begin{array}{l}g=22.14 \pm 0.10 \\
r=21.23 \pm 0.10\end{array}$ & $\begin{array}{l}g=21.55 \pm 0.05 \\
r=20.01 \pm 0.03 \\
i=18.35 \pm 0.03 \\
z=17.46 \pm 0.04\end{array}$ & $\begin{array}{l}\cdots \\
\ldots \\
\cdots \\
\ldots\end{array}$ & M5 & 315 \\
\hline PSO J130.6460 + 45.2130 & $08^{\mathrm{h}} 42^{\mathrm{m}} 35^{\mathrm{s}} .063$ & $+45^{\circ} 12^{\prime} 47^{\prime \prime} .07$ & 2009 Dec 14 & $\begin{array}{l}g=22.07 \pm 0.05 \\
r=22.05 \pm 0.05\end{array}$ & $\begin{array}{c}g \gtrsim 25.28 \\
r \gtrsim 25.31 \\
i=22.56 \pm 0.04 \\
z=21.00 \pm 0.02\end{array}$ & $\begin{array}{l}\cdots \\
\cdots \\
\cdots \\
\cdots\end{array}$ & M9 & 290 \\
\hline PSO J162.3497 + 56.6609 & $10^{\mathrm{h}} 49^{\mathrm{m}} 23^{\mathrm{s}} .934$ & $+56^{\circ} 39^{\prime} 39^{\prime \prime} 57$ & 2011 Apr 29 & $\begin{array}{l}g=18.48 \pm 0.02 \\
r=18.93 \pm 0.05\end{array}$ & $\begin{array}{l}g=23.62 \pm 0.07 \\
r=22.19 \pm 0.04 \\
i=19.96 \pm 0.01 \\
z=18.78 \pm 0.04\end{array}$ & $\begin{array}{l}g=23.45 \pm 0.23 \\
r=22.35 \pm 0.16 \\
i=19.97 \pm 0.03 \\
z=18.69 \pm 0.04\end{array}$ & M7.5 & 220 \\
\hline PSO J164.3814 + 58.3011 & $10^{\mathrm{h}} 57^{\mathrm{m}} 31^{\mathrm{s}} .557$ & $+58^{\circ} 18^{\prime} 04^{\prime \prime} .12$ & 2011 Apr 14 & $\begin{array}{l}g=19.08 \pm 0.01 \\
r=21.08 \pm 0.04\end{array}$ & $\begin{array}{c}g \gtrsim 25.25 \\
r=24.07 \pm 0.10 \\
i=21.72 \pm 0.02 \\
z=20.20 \pm 0.01\end{array}$ & $\begin{array}{c}\ldots \\
r=23.51 \pm 0.31 \\
i=21.45 \pm 0.08 \\
z=19.79 \pm 0.07\end{array}$ & M8.5 & 330 \\
\hline PSO J184.0215 + 47.4267 & $12^{\mathrm{h}} 16^{\mathrm{m}} 05^{\mathrm{s}} .180$ & $+47^{\circ} 25^{\prime} 36^{\prime \prime} 24$ & 2010 Jun 6 & $\begin{array}{l}g=20.61 \pm 0.03 \\
r=21.10 \pm 0.10\end{array}$ & $\begin{array}{l}g=21.88 \pm 0.05 \\
r=20.47 \pm 0.03 \\
i=18.96 \pm 0.04 \\
z=18.14 \pm 0.05\end{array}$ & $\begin{array}{l}g=21.83 \pm 0.06 \\
r=20.40 \pm 0.03 \\
i=18.87 \pm 0.01 \\
z=18.05 \pm 0.02\end{array}$ & M4.5 & 600 \\
\hline PSO J184.6519+48.1834 & $12^{\mathrm{h}} 18^{\mathrm{m}} 36^{\mathrm{s}} .472$ & $+48^{\circ} 11^{\prime} 00^{\prime \prime} 41$ & 2010 Feb 9 & $\begin{array}{l}g=18.96 \pm 0.01 \\
r=19.60 \pm 0.02\end{array}$ & $\begin{array}{l}g=20.12 \pm 0.02 \\
r=18.80 \pm 0.02 \\
i=17.32 \pm 0.04 \\
z=16.66 \pm 0.04\end{array}$ & $\begin{array}{l}g=20.18 \pm 0.02 \\
r=18.75 \pm 0.01 \\
i=17.34 \pm 0.01 \\
z=16.57 \pm 0.01\end{array}$ & M4.5 & 280 \\
\hline PSO J185.4857+ 47.2586 & $12^{\mathrm{h}} 21^{\mathrm{m}} 56^{\mathrm{s}} .583$ & $+47^{\circ} 15^{\prime} 31^{\prime \prime} 14$ & 2011 Jun 7 & $\begin{array}{l}g=22.21 \pm 0.10 \\
r=22.33 \pm 0.10\end{array}$ & $\begin{array}{c}g \gtrsim 25.10 \\
r \gtrsim 24.95 \\
i=24.25 \pm 0.20 \\
z=22.85 \pm 0.11\end{array}$ & $\begin{array}{l}\cdots \\
\cdots \\
\cdots \\
\cdots\end{array}$ & M8 & 1160 \\
\hline PSO J211.8195 + 52.9681 & $14^{\mathrm{h}} 07^{\mathrm{m}} 16^{\mathrm{s}} .681$ & $+52^{\circ} 58^{\prime} 05^{\prime \prime} .27$ & 2011 Jun 7 & $\begin{array}{l}g=20.60 \pm 0.02 \\
r=20.62 \pm 0.02\end{array}$ & $\begin{array}{l}g=21.95 \pm 0.04 \\
r=20.63 \pm 0.02 \\
i=19.15 \pm 0.02 \\
z=18.39 \pm 0.02\end{array}$ & $\begin{array}{l}g=21.92 \pm 0.08 \\
r=20.57 \pm 0.04 \\
i=19.11 \pm 0.02 \\
z=18.27 \pm 0.03\end{array}$ & M4.5 & 650 \\
\hline PSO J214.7904 + 53.6886 & $14^{\mathrm{h}} 19^{\mathrm{m}} 09^{\mathrm{s}} .715$ & $+53^{\circ} 41^{\prime} 18^{\prime \prime} .99$ & 2010 Feb 6 & $\begin{array}{l}g=21.60 \pm 0.08 \\
r=20.86 \pm 0.08\end{array}$ & $\begin{array}{l}g=20.11 \pm 0.02 \\
r=18.71 \pm 0.02 \\
i=17.10 \pm 0.02 \\
z=16.38 \pm 0.04\end{array}$ & $\begin{array}{l}g=20.22 \pm 0.02 \\
r=18.71 \pm 0.01 \\
i=17.10 \pm 0.01 \\
z=16.23 \pm 0.01\end{array}$ & M5 & 220 \\
\hline PSO J334.5602-0.8479 & $22^{\mathrm{h}} 18^{\mathrm{m}} 14^{\mathrm{s}} .455$ & $-00^{\circ} 50^{\prime} 52^{\prime \prime} .77$ & $2011 \mathrm{Jul} 31$ & $\begin{array}{l}g=22.45 \pm 0.08 \\
r=22.40 \pm 0.10\end{array}$ & $\begin{array}{l}g=22.67 \pm 0.04 \\
r=21.23 \pm 0.02 \\
i=19.67 \pm 0.02 \\
z=18.84 \pm 0.02\end{array}$ & $\begin{array}{l}g=22.78 \pm 0.17 \\
r=21.20 \pm 0.05 \\
i=19.66 \pm 0.02 \\
z=18.87 \pm 0.04\end{array}$ & M4.5 & 690 \\
\hline PSO J334.7536+ 0.8769 & $22^{\mathrm{h}} 19^{\mathrm{m}} 00^{\mathrm{s}} .865$ & $+00^{\circ} 52^{\prime} 37^{\prime \prime} .11$ & 2010 Sep 4 & $\begin{array}{l}g=21.93 \pm 0.06 \\
r=21.84 \pm 0.06\end{array}$ & $\begin{array}{l}g=23.32 \pm 0.06 \\
r=21.77 \pm 0.02 \\
i=20.02 \pm 0.01 \\
z=19.12 \pm 0.01\end{array}$ & $\begin{array}{l}g=22.94 \pm 0.20 \\
r=21.76 \pm 0.09 \\
i=19.99 \pm 0.03 \\
z=19.09 \pm 0.06\end{array}$ & M5.5 & 570 \\
\hline
\end{tabular}

Notes. Properties of the fast transients that exhibit quiescent counterparts, as well as multi-band photometry of the counterparts. We find that all 11 transients arise from $\mathrm{M}$ dwarfs, and provide the inferred spectral types and distances of these sources.

$\left(g_{\mathrm{P} 1} r_{\mathrm{P} 1} i_{\mathrm{P} 1} z_{\mathrm{P} 1}\right)$ to typical limits of $\gtrsim 24.5-25 \mathrm{mag}$. These sources are in principle a promising population of distant extragalactic transients with undetected host galaxies. We utilize a combination of color information, the 33 minute time-span of the $g_{\mathrm{P} 1} r_{\mathrm{P} 1}$ observations, and the sky locations to investigate the nature of these sources. We first note that all eight sources span a narrow color range with $(g-r)_{\mathrm{P} 1} \approx 0.55-0.8 \mathrm{mag}$ (Figure 3 and Table 2), which is typical of main-belt asteroids (e.g., Ivezić et al. 2001). This interpretation naturally explains the lack of quiescent counterparts in the template images. We further test this scenario by comparing the astrometric positions of each transient in the $g_{\mathrm{P} 1}$ and $r_{\mathrm{P} 1}$ images. In Figure 4, we show the distribution of positional shifts for the eight sources compared to unresolved field sources in the same images. The median offset for field sources is about 53 mas (with a standard deviation of about 31 mas), indicative of the astrometric alignment precision of the MDS images. On the other hand, the eight transients exhibit shifts of 11-340 mas (bounded by our initial cut of $\lesssim 0$.'35 shift; Section 2.2), with a median value of about 230 mas. This is well in excess of the point source population, 
Table 2

Fast Optical Transients Lacking Quiescent Counterparts

\begin{tabular}{|c|c|c|c|c|c|c|c|}
\hline OBJID & $\begin{array}{l}\text { R.A. } \\
(\mathrm{h} \mathrm{m} \mathrm{s})\end{array}$ & $\begin{array}{l}\text { Decl. } \\
\left({ }^{\circ \prime \prime \prime}\right)\end{array}$ & $\begin{array}{c}\ell \\
(\mathrm{deg})\end{array}$ & $\begin{array}{c}b \\
(\mathrm{deg})\end{array}$ & UT Date & $\begin{array}{c}g_{\mathrm{P} 1} \\
(\mathrm{AB} \text { mag) }\end{array}$ & $\begin{array}{c}r_{\mathrm{P} 1} \\
(\mathrm{AB} \text { mag) }\end{array}$ \\
\hline PSO J149.0662 + 1.8129 & $09^{\mathrm{h}} 56^{\mathrm{m}} 15^{\mathrm{s}} .886$ & $+01^{\circ} 48^{\prime} 46^{\prime \prime} 46$ & 150.5546 & -10.0989 & 2011 Apr 23 & $22.73 \pm 0.08$ & $22.14 \pm 0.06$ \\
\hline PSO J149.2788+3.3718 & $09^{\mathrm{h}} 57^{\mathrm{m}} 06^{\mathrm{s}} .910$ & $+03^{\circ} 22^{\prime} 18^{\prime \prime} .37$ & 150.2096 & -8.5629 & 2011 Apr 23 & $19.78 \pm 0.02$ & $19.25 \pm 0.03$ \\
\hline PSO J149.3234 + 2.6863 & $09^{\mathrm{h}} 57^{\mathrm{m}} 17^{\mathrm{s}} .622$ & $+02^{\circ} 41^{\prime} 10^{\prime \prime} 51$ & 150.4922 & -9.1906 & 2011 Apr 23 & $20.44 \pm 0.03$ & $19.90 \pm 0.03$ \\
\hline PSO J333.1738+ 0.4535 & $22^{\mathrm{h}} 12^{\mathrm{m}} 41^{\mathrm{s}} .723$ & $+00^{\circ} 27^{\prime} 12^{\prime \prime} .69$ & 335.2762 & +10.7642 & 2011 Oct 24 & $22.13 \pm 0.06$ & $21.47 \pm 0.04$ \\
\hline PSO J333.9157-0.6311 & $22^{\mathrm{h}} 15^{\mathrm{m}} 39^{\mathrm{s}} .776$ & $-00^{\circ} 37^{\prime} 51^{\prime \prime} .89$ & 335.5803 & +9.4847 & 2010 Oct 10 & $21.53 \pm 0.06$ & $20.96 \pm 0.03$ \\
\hline PSO J352.2672 + 1.2637 & $23^{\mathrm{h}} 29^{\mathrm{m}} 04^{\mathrm{s}} .139$ & $-01^{\circ} 15^{\prime} 49^{\prime \prime} .35$ & 352.3995 & +1.9068 & 2010 Nov 3 & $21.35 \pm 0.04$ & $20.55 \pm 0.02$ \\
\hline PSO J352.5520+0.8004 & $23^{\mathrm{h}} 30^{\mathrm{m}} 12^{\mathrm{s}} .469$ & $+00^{\circ} 48^{\prime} 01^{\prime \prime} .49$ & 353.4772 & +3.6909 & 2010 Nov 3 & $22.40 \pm 0.10$ & $21.67 \pm 0.07$ \\
\hline PSO J352.5968-0.4471 & $23^{\mathrm{h}} 30^{\mathrm{m}} 23^{\mathrm{s}} \cdot 229$ & $-00^{\circ} 26^{\prime} 49^{\prime \prime} 64$ & 353.0249 & +2.5271 & 2010 Oct 31 & $19.59 \pm 0.02$ & $18.79 \pm 0.02$ \\
\hline
\end{tabular}

Notes. Properties of the fast transients that lack quiescent counterparts. We find that all eight transients exhibit the colors and expected ecliptic coordinates of main-belt asteroids near the stationary point of their orbits.

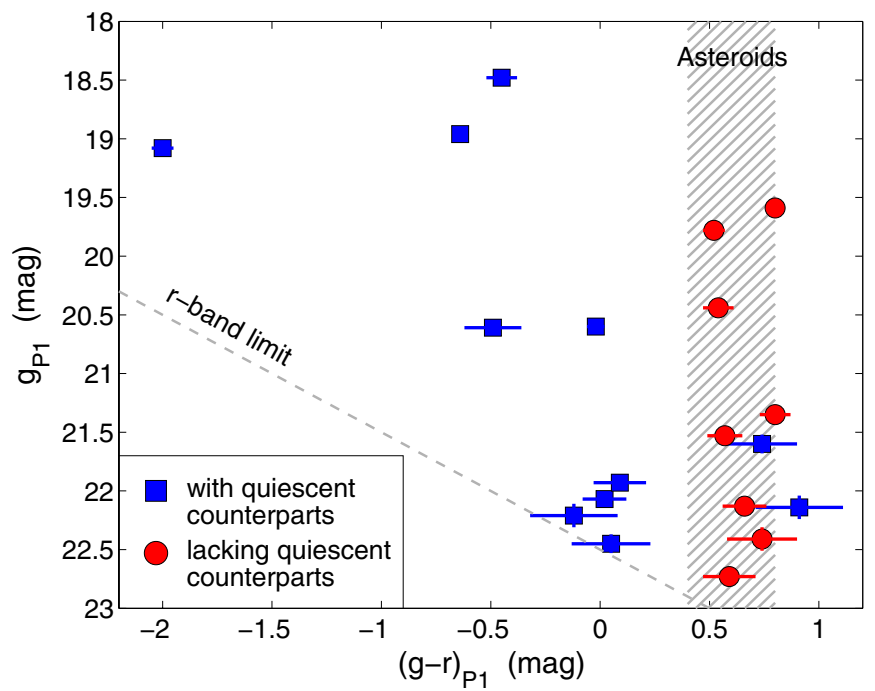

Figure 3. Color-magnitude diagram for the fast transients discovered in our PS1/MDS search. The sources are divided into those with detected quiescent counterparts in the deep template images (blue squares), and those lacking counterparts to limits of $g_{\mathrm{P} 1} r_{\mathrm{P} 1} i_{\mathrm{P} 1} z_{\mathrm{P} 1} \gtrsim 24.5-25 \mathrm{mag}$ (red circles). The dashed line marks the $r_{\mathrm{P} 1}$ limit of our search (the region below the line is inaccessible to the survey). The hatched region marks the expected $(g-r)_{\mathrm{P} 1}$ color range for asteroids. All of the fast transients lacking quiescent counterparts reside in this color range.

(A color version of this figure is available in the online journal.)

and a Kolmogorov-Smirnov (K-S) test gives a $p$-value of only $1.1 \times 10^{-4}$ for the null hypothesis that the positional offsets of the eight transients and the field sources are drawn from the same underlying distribution. This clearly indicates that the eight transients lacking quiescent counterparts exhibit larger than average astrometric shifts, supporting their identification as asteroids. We further inspect the $g_{\mathrm{P} 1}$ and $r_{\mathrm{P} 1}$ light curves of the eight transients and find that none exhibit variability larger than the photometric uncertainties (e.g., Figure 2).

Finally, we note that all eight sources are located in the three MDS fields (MD04, MD09, and MD10) that are positioned within $\pm 10^{\circ}$ of the ecliptic plane (Table 2). In particular, the asteroids were discovered in these fields on dates concentrated at solar elongation values of about $130^{\circ}$, at which main-belt asteroids go through a stationary point with negligible apparent motion. We therefore conclude based on their colors, astrometric motions, light-curve behavior, and ecliptic coordinates that the eight fast transients lacking quiescent counterparts are simply main-belt asteroids near the stationary point of their orbit.

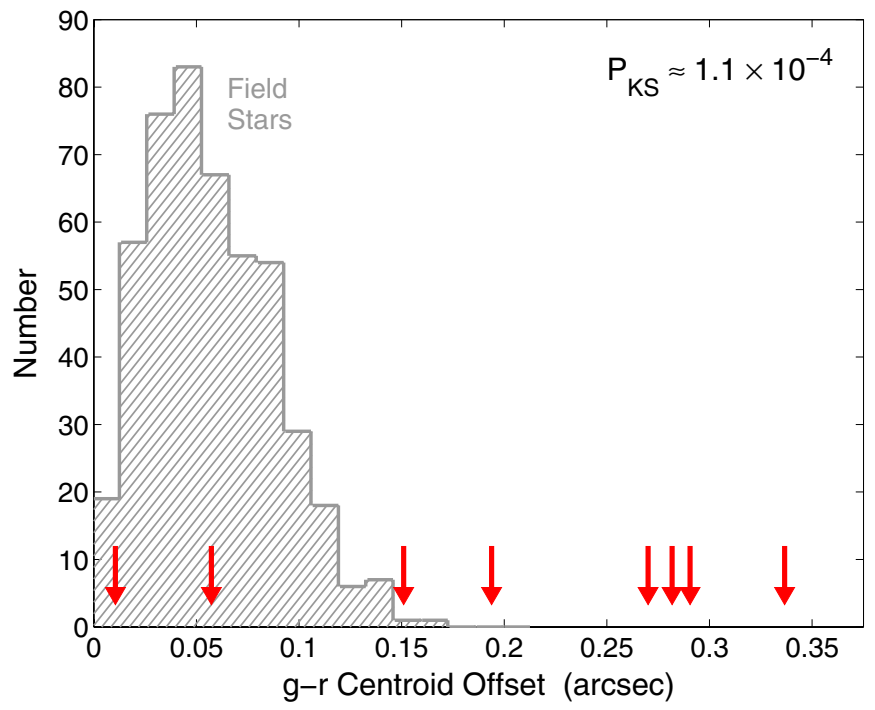

Figure 4. Astrometric shift between the $g_{\mathrm{P} 1}$ and $r_{\mathrm{P} 1}$ centroids for the fast transients lacking quiescent counterparts (red arrows) in comparison to the shifts for field stars from the same images (gray hatched histogram). The median positional shift for the field stars, which indicates the typical astrometric uncertainty of the MDS images, is about 53 mas. The fast transients lacking quiescent counterparts exhibit generally larger shifts of up to $\approx 340$ mas (the limit allowed by our search), with a median of 230 mas. A K-S test indicates a $p$-value of only $1.1 \times 10^{-4}$ for the null hypothesis that the two populations are drawn from the same underlying distribution.

(A color version of this figure is available in the online journal.)

\subsection{Fast Transients with Detected Quiescent Counterparts}

We now turn to the 11 fast transients that exhibit quiescent counterparts in some or all of the deep MDS template images. In all cases we find that the counterparts are unresolved (with a typical seeing of about $1^{\prime \prime}$ ) and have red colors that are indicative of M-dwarf stars. Photometry of the quiescent counterparts from the PS1/MDS templates, and from SDSS when available, is summarized in Table 1. Using these magnitudes, we determine the spectral type of each counterpart by comparing to the SDSS colors of M dwarfs (West et al. 2011); from sources with both PS1 and SDSS photometry, we infer color transformations of $(g-r)_{\mathrm{P} 1} \approx 0.94 \times(g-r)_{\mathrm{SDSS}}$ and $(i-z)_{\mathrm{P} 1} \approx 0.93 \times(i-z)_{\mathrm{SDSS}}$ to account for the difference between the $g_{\mathrm{P} 1}$ and $z_{\mathrm{P} 1}$ filters compared to the $g_{\text {SDSS }}$ and $z_{\text {SDSS }}$ filters (Section 2.1). The results are shown in Figure 5 indicating that seven counterparts have spectral types of about M4-M5, while the remaining four counterparts have spectral types of M7-M9 (see Table 1 for the inferred spectral types). We further infer the distances to these $\mathrm{M}$ dwarfs 


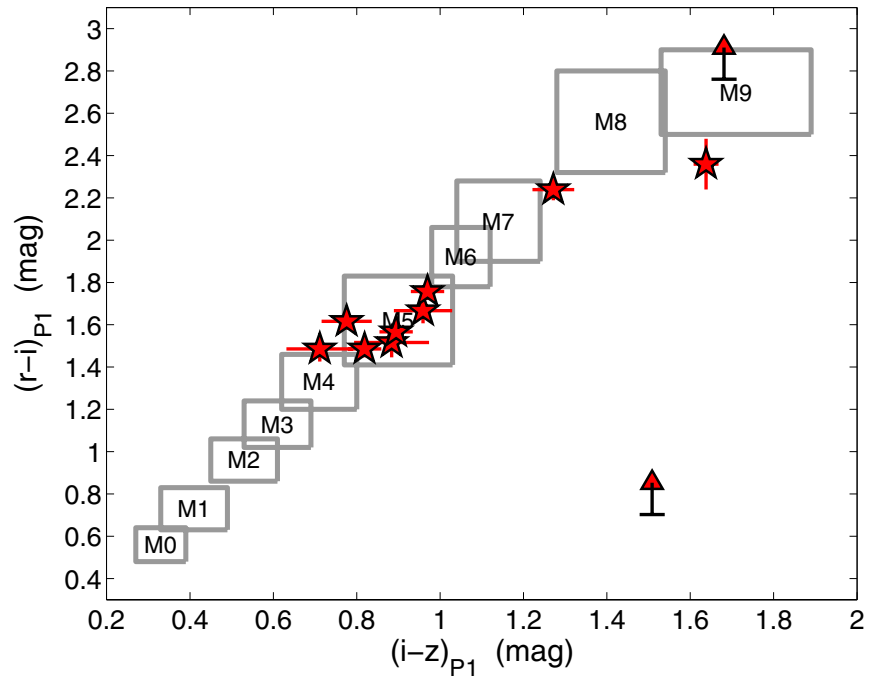

Figure 5. Color-color phase space for the quiescent counterparts of all 11 fast transients with a detected counterpart (stars; arrows indicate limits). Also shown are the color ranges for M0-M9 dwarf stars (West et al. 2011). All of the detected counterparts track the M-dwarf sequence, with seven sources exhibiting the colors of M4-M5 dwarfs and four sources exhibiting colors typical of M7-M9 dwarfs.

(A color version of this figure is available in the online journal.)

using their associated absolute magnitudes (Bochanski et al. 2011) and find $d \approx 0.2-1.2 \mathrm{kpc}$ (Table 1 ).

Thus, as in previous fast transient searches, all 11 fast transients with quiescent counterparts in our survey are M dwarf flares. Using the flare magnitudes and inferred spectral types, we compare the resulting flare and bolometric luminosities in Figure 6 . We find that the flares span a luminosity ${ }^{10}$ range of $L_{f, g} \approx(6-150) \times 10^{28} \mathrm{erg} \mathrm{s}^{-1}$ and $L_{f, r} \approx(4-80) \times 10^{28} \mathrm{erg} \mathrm{s}^{-1}$, with no apparent dependence on spectral type. However, since the bolometric luminosity declines from about $3.3 \times 10^{31} \mathrm{erg} \mathrm{s}^{-1}$ at spectral type M4 to about $1.3 \times 10^{30} \mathrm{erg} \mathrm{s}^{-1}$ at spectral type M9, the relative flare luminosities increase with later spectral type. We find relative flare luminosities of $\approx 0.006-0.07 L_{\mathrm{bol}}$ at $\sim \mathrm{M} 5$, and larger values of $\approx 0.03-1 L_{\text {bol }}$ at M8-M9.

It is instructive to compare the properties of the flares and $M$ dwarfs uncovered in our blind fast transients search to those from targeted M-dwarf variability studies. In particular, Kowalski et al. (2009) searched for flares from 50,130 preselected M0-M6 dwarfs in the SDSS Stripe 82 and found 271 flares, with an apparent increase in the flare rate with later spectral type. This may explain the lack of M0-M4 dwarfs in our relatively small sample. For the M4-M6 dwarfs, Kowalski et al. (2009) find a mean flare amplitude of $\Delta u \approx 1.5 \mathrm{mag}$, comparable to our mean value of $\Delta g \approx 1.1 \mathrm{mag}$, when taking into account that $M$ dwarfs' flares are generally brighter in $u$ band than in $g$ band due a typical temperature of $\sim 10^{4} \mathrm{~K}$. The flare luminosities for the Stripe 82 M4-M6 dwarfs are $L_{f, u} \approx(2-100) \times 10^{28} \mathrm{erg} \mathrm{s}^{-1}$, again comparable to the $g_{\mathrm{P}^{-}}$ band luminosities of the $\sim$ M5 dwarfs in our sample. We note that there are no M7-M9 dwarfs in the Stripe 82 sample.

Kowalski et al. (2009) also found that for M4-M6 dwarfs there is a strong dependence of the flare rate on vertical distance from the Galactic plane; namely, the fraction of time in which

\footnotetext{
10 We determine the luminosity by integrating the spectral luminosity over the widths of the $g_{\mathrm{P} 1}$ and $r_{\mathrm{P} 1}$ filters, with $\delta v \approx 1.287 \times 10^{14} \mathrm{~Hz}$ and $\approx 7.721 \times 10^{13} \mathrm{~Hz}$, respectively.
}

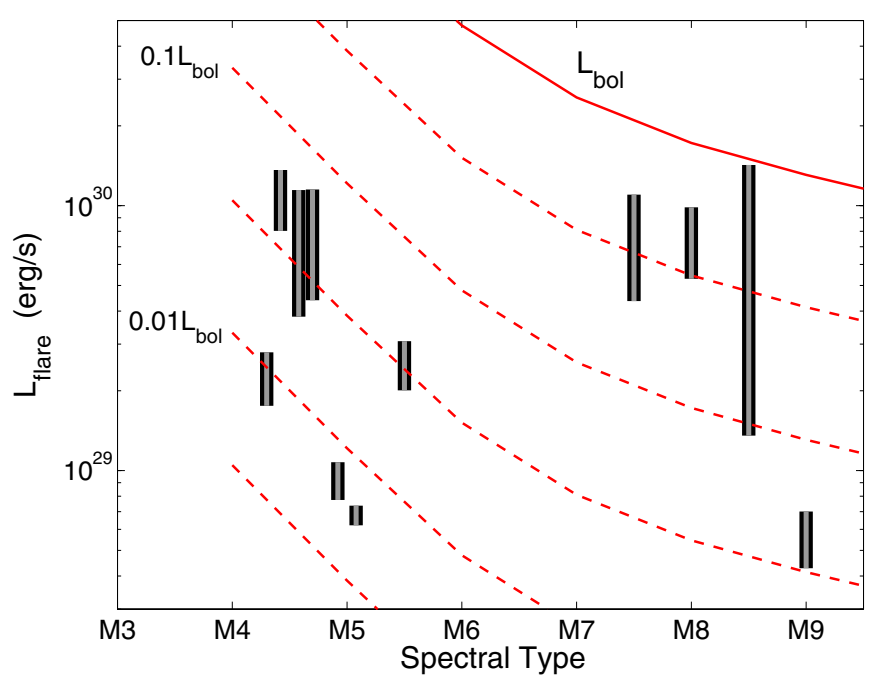

Figure 6. Luminosities of the fast transients (flares) associated with M-dwarf counterparts (vertical bars) as a function of source spectral type. The luminosity range for each source is defined by the $g_{\mathrm{P} 1}$ and $r_{\mathrm{P} 1}$ detections. The solid red line marks the bolometric luminosity as a function of spectral type, while the dashed lines mark fractions of the bolometric luminosity as indicated in the figure. We find that the flares from the $\sim$ M5 sources have a range of $\approx 0.006-0.07 L_{\text {bol }}$, while the flares from the M7-M9 dwarfs are relatively larger, with $\approx 0.03-1 L_{\text {bol }}$. (A color version of this figure is available in the online journal.)

a star flares decreases by about an order of magnitude over a vertical distance range of about $50-150$ pc. The M4-M6 dwarfs in our sample are all located at larger vertical distances of $\approx 190-560 \mathrm{pc}$, with a mean of about $390 \mathrm{pc}$, suggesting that the decline in flaring activity with vertical distance from the Galactic plane may not be as steep as previously inferred. Moreover, the Stripe 82 data exhibit a trend of steeper decline in the flare rate as a function of vertical distance with increasing spectral type (Kowalski et al. 2009), while here we find that the M7-M9 dwarfs have a similar mean vertical distance from the Galactic plane to the $\sim$ M5 dwarfs. Clearly, a more systematic search for M-dwarf flares in the PS1/MDS is required to study these trends. In particular, it is likely that we have missed some flares due to the requirement of no additional variability within $\mathrm{a} \pm 5$ night window around each detection (Section 2.2).

\section{LIMITS ON THE RATE OF EXTRAGALACTIC FAST OPTICAL TRANSIENTS}

The 19 fast transients uncovered by our search cleanly divide into two categories: (1) main-belt asteroids near the stationary point of their orbits (Section 3.1) and (2) flares from M-dwarf stars (Section 3.2). Neither category is unexpected given that our search is based on consecutive $g_{\mathrm{P} 1} r_{\mathrm{P} 1}$ detections with a timespan of about $0.5 \mathrm{hr}$. M-dwarf flares typically exhibit blue colors indicative of $T \sim 10^{4} \mathrm{~K}$, with timescales of minutes to hours, and are thus ubiquitous in searches that utilize rapid observations in the ultraviolet (Welsh et al. 2005, 2006) or blue optical bands (Becker et al. 2004; Kulkarni \& Rau 2006; Rau et al. 2008). Similarly, our requirement of two consecutive detections within $\sim 0.5 \mathrm{hr}$, with non-detections on preceding or subsequent nights, is effective at capturing asteroids near the stationary point of their orbits (i.e., at solar elongations of about $130^{\circ}$ for main-belt asteroids).

Since we account for all 19 fast transients as solar system or Galactic in origin, we can place a robust upper limit on the rate of extragalactic FOTs. While we could in principle detect 
Table 3

Surveys for Fast Optical Transients with a Timescale of $\sim 0.5 \mathrm{hr}$

\begin{tabular}{|c|c|c|c|c|c|c|c|}
\hline Survey & $\begin{array}{l}\text { Areal Exposure } \\
\quad\left(\operatorname{deg}^{2} \text { day }\right)\end{array}$ & Limiting Magnitude & $\begin{array}{c}R_{\mathrm{FOT}} \\
\left(\mathrm{deg}^{-2} \text { day }^{-1}\right)\end{array}$ & $\begin{array}{c}R_{\mathrm{FOT}}(-10 \mathrm{mag}) \\
\left(\mathrm{Mpc}^{-3} \mathrm{yr}^{-1}\right)\end{array}$ & $\begin{array}{c}R_{\mathrm{FOT}}(-14 \mathrm{mag}) \\
\left(\mathrm{Mpc}^{-3} \mathrm{yr}^{-1}\right)\end{array}$ & $\begin{array}{c}R_{\mathrm{FOT}}(-24 \mathrm{mag}) \\
\left(\mathrm{Mpc}^{-3} \mathrm{yr}^{-1}\right)\end{array}$ & References \\
\hline PS1/MDS & 40.4 & 22.5 & $\lesssim 0.12$ & $\lesssim 13$ & $\lesssim 0.05$ & $\lesssim 1 \times 10^{-6}$ & This paper \\
\hline DLS & 1.1 & 23.8 & $\leq 6.5$ & $\lesssim 1.3 \times 10^{2}$ & $\stackrel{\sim}{\leq} 0.5$ & $\leq 6 \times 10^{-5}$ & Becker et al. (2004) \\
\hline Fornax & 1.9 & 21.3 & $\lesssim 3.3$ & $\lesssim 2.0 \times 10^{3}$ & $\lesssim 7.8$ & $\lesssim 3 \times 10^{-5}$ & Rau et al. (2008) \\
\hline ROTSE-III & 635 & 17.5 & $\widetilde{<0.005}$ & $\sim$ & $\sim \ldots$ & $\stackrel{\sim}{\leqslant} 6 \times 10^{-6}$ & Rykoff et al. (2005) \\
\hline MASTER & $\ldots$ & 17.5 & $\lesssim 0.003$ & $\ldots$ & $\ldots$ & $\stackrel{\lesssim}{\lesssim} 4 \times 10^{-6}$ & Lipunov et al. (2007) \\
\hline \multirow[t]{2}{*}{ LSST } & 62 & 23.5 & $\lesssim 0.07$ & $\lesssim 2$ & $\leq 8 \times 10^{-3}$ & $\lesssim 5 \times 10^{-7}$ & . \\
\hline & $1.6 \times 10^{4}$ & 23.5 & $\lesssim 3 \times 10^{-4}$ & $\lesssim 9 \sim 10^{-3}$ & $\lesssim 3 \times 10^{-5}$ & $\stackrel{\approx}{\approx} 2 \times 10^{-9}$ & $\ldots$ \\
\hline
\end{tabular}

Note. Survey parameters and resulting limits on the projected sky rates and volumetric rates of extragalactic fast optical transients with a timescale of $\sim 0.5$ hr.

a sufficiently bright transient with a timescale as short as about four minutes, corresponding to a detection in only the final exposure in one filter and the first exposure in the second filter, a more reasonable timescale probed by our search is about $0.5 \mathrm{hr}$, the time-span of a full $g_{\mathrm{P} 1} r_{\mathrm{P} 1}$ exposure sequence (e.g., Figure 2). Similarly, our selection criteria could in principle accommodate transients with durations as long as $\sim 2$ day, but for the bulk of the search the maximum timescale is $\lesssim 1$ day. Thus, we consider our search to place limits on fast transients spanning about $0.5 \mathrm{hr}$ to 1 day. In the discussion below, we provide upper limits for the upper and lower bounds of the timescale distribution.

The total areal exposure of our survey for a timescale of $0.5 \mathrm{hr}$ is $E_{A} \approx 40.4 \mathrm{deg}^{2}$ day, while for a timescale of $\sim 1$ day it is correspondingly longer, $E_{A} \approx 1940 \mathrm{deg}^{2}$ day. Thus, we can place a $95 \%$ confidence limit $(\lesssim 3$ events) on the sky-projected rate of

$$
R_{\mathrm{FOT}}=N / \epsilon^{2} E_{A},
$$

where we estimate the detection efficiency per filter at $\epsilon \approx$ 0.8 based on the overall search for transients in the MDS fields. Thus, for a timescale of $0.5 \mathrm{hr}$ we place a limit of $R_{\text {FOT }} \lesssim 0.12 \mathrm{deg}^{-2}$ day $^{-1}$, while for a timescale of $\sim 1$ day it is $R_{\text {FOT }} \lesssim 2.4 \times 10^{-3} \mathrm{deg}^{-2}$ day $^{-1}$ (see Table 3 ).

Our limit on fast transients with a $0.5 \mathrm{hr}$ timescale improves on existing searches with similar limiting magnitudes by a factor of 30-50 (Figure 7) thanks to the much larger effective areal exposure. DLS ( $E_{A} \approx 1.1 \mathrm{deg}^{2}$ day) placed an upper limit of $R_{\text {FOT }} \lesssim 6.5$ events $\mathrm{deg}^{-2}$ day $^{-1}$ for transients with a timescale of about $0.36 \mathrm{hr}$ (Becker et al. 2004), while the Fornax galaxy cluster search $\left(E_{A} \approx 1.9 \mathrm{deg}^{2}\right.$ day) placed a limit of $R_{\mathrm{FOT}} \lesssim$ $3.3 \mathrm{deg}^{-2} \mathrm{day}^{-1}$ for transients with a timescale of about $0.55 \mathrm{hr}$ (Rau et al. 2008). The ROTSE-III search $\left(E_{A} \approx 635 \mathrm{deg}^{2}\right.$ day) placed a limit of $R_{\mathrm{FOT}} \lesssim 5 \times 10^{-3} \mathrm{deg}^{-2}$ day $^{-1}$ for transients with a timescale of about $0.5 \mathrm{hr}$, but was significantly shallower (Rykoff et al. 2005).

The sky-projected rates do not take into account the difference in limiting magnitudes between various searches. Our survey limiting magnitude is about $1.3 \mathrm{mag}$ shallower than the DLS search, but about 1.2 mag deeper than the Fornax cluster search, and about 5 mag deeper than the ROTSE-III search. This information is summarized in Table 3, and shown in the relevant three-dimensional phase space of survey limiting magnitude, timescale, and sky-projected rate limit in Figure 7.

The survey depth impacts the inferred limits on volumetric rates. For example, for a population of fast transients with a fiducial absolute magnitude of -10 (comparable to the most luminous known novae; see Section 5), the limits on the

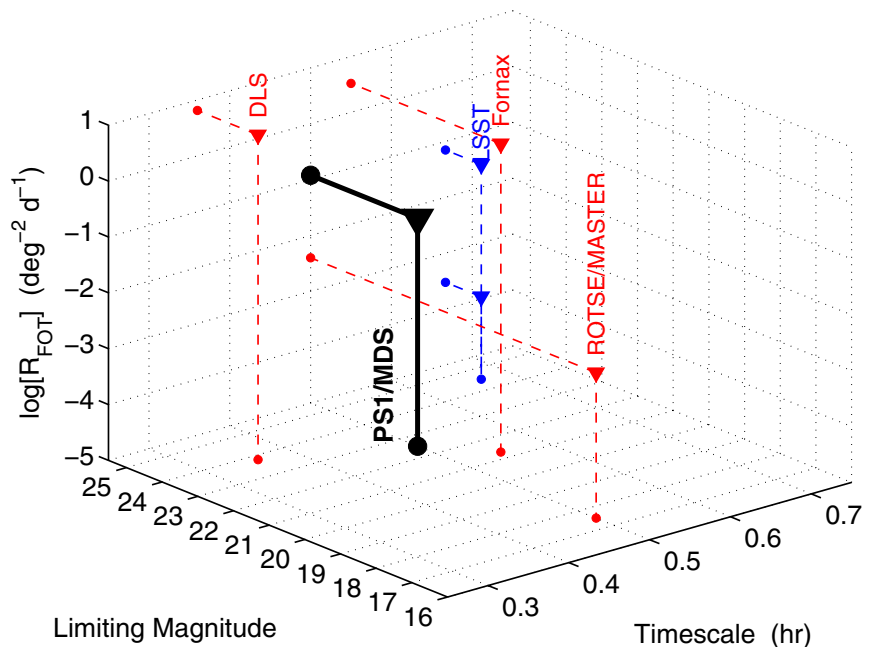

Figure 7. Limits on the sky-projected rate of extragalactic fast optical transients as a function of timescale and survey limiting magnitude. Shown are the limits from our survey (black) and from the literature (red; Becker et al. 2004; Rykoff et al. 2005; Lipunov et al. 2007; Rau et al. 2008). The much larger effective areal exposure of our survey provides constraints that are about 30-50 times deeper than previous surveys with similar limiting magnitudes. Also shown are the expected limits from the LSST main survey (blue) for one night (top limit) and one year (bottom limit) of observations.

(A color version of this figure is available in the online journal.)

volumetric rates ${ }^{11}$ on a timescale of $0.5 \mathrm{hr}$ are $\lesssim 13 \mathrm{Mpc}^{-3} \mathrm{yr}^{-1}$ for our survey, $\lesssim 1.3 \times 10^{2} \mathrm{Mpc}^{-3} \mathrm{yr}^{-1}$ for DLS, and $\lesssim 2.0 \times$ $10^{3} \mathrm{Mpc}^{-3} \mathrm{yr}^{-1}$ for Fornax. The small volume probed by the ROTSE-III search for transients with $-10 \mathrm{mag}(d \approx 3 \mathrm{Mpc})$ provides no real insight on the extragalactic population. For a fiducial absolute magnitude of -14 (comparable to the least luminous supernovae), the limits are $\lesssim 0.05$ (PS1/MDS), $\lesssim 0.5$ (DLS), and $\lesssim 7.8$ (Fornax) $\mathrm{Mpc}^{-3} \mathrm{yr}^{-1}$; at this peak magnitude, the maximal detection distances are large enough to uniformly sample the galaxy distribution (about $200 \mathrm{Mpc}$ for our search, $360 \mathrm{Mpc}$ for DLS, and $115 \mathrm{Mpc}$ for Fornax). Finally, for a fiducial absolute magnitude of -24 (intermediate between long and short gamma-ray burst (GRB) afterglows; see Section 5), the volumetric rate limits are $\lesssim 10^{-6}(\mathrm{PS} 1 / \mathrm{MDS}), \lesssim 6 \times 10^{-5}$

\footnotetext{
11 We assume a uniform underlying distribution of galaxies within the volume probed by each search, which is not strictly the case for the maximum distances associated with a peak magnitude of -10: $32 \mathrm{Mpc}$ for our search, $56 \mathrm{Mpc}$ for DLS, and $18 \mathrm{Mpc}$ for Fornax. With the exception of the Fornax search, which was centered on the Fornax galaxy cluster at $d \approx 16 \mathrm{Mpc}$, this indicates that the actual limits from our survey and from DLS are subject to the underlying non-uniform galaxy distribution.
} 
(DLS), and $\lesssim 3 \times 10^{-5}$ (Fornax) $\mathrm{Mpc}^{-3} \mathrm{yr}^{-1}$. The various limits are summarized in Table 3.

We note that the inferred limits at -10 and -14 mag are substantially higher than the volumetric rate of supernovae, $\approx 10^{-4} \mathrm{Mpc}^{-3} \mathrm{yr}^{-1}$ (Li et al. 2011), and novae ${ }^{12}$, $\approx 0.1 \mathrm{Mpc}^{-3} \mathrm{yr}^{-1}$; similarly, the limits at $-24 \mathrm{mag}$ are at least two orders of magnitude larger than the on-axis GRB rate. This indicates that any source population of extragalactic FOTs with a timescale of $0.5 \mathrm{hr}$ would have to be much more abundant than nova, supernova, or GRB progenitors, or produce multiple $\left(\sim 10^{2}-10^{3}\right)$ events per progenitor system to be detected with current surveys.

Utilizing the $B$-band luminosity density in the local universe $\left(\approx 1.4 \times 10^{8} L_{B, \odot} \mathrm{Mpc}^{-3}\right)$, the upper limits on the volumetric rates at $-10 \mathrm{mag}$ can be recast as $\lesssim 9.2 \times 10^{2}$ (PS1/MDS) and $\lesssim 9.3 \times 10^{3}$ (DLS) $\mathrm{yr}^{-1}$ per $10^{10} L_{B, \odot}$; for the Fornax survey, which targeted a galaxy cluster environment, the limit is $\lesssim 350 \mathrm{yr}^{-1}$ per $10^{10} L_{B, \odot}$ (Rau et al. 2008). For a fiducial brightness of $-14 \mathrm{mag}$, the limits are $\lesssim 3.6$ (PS1/MDS), $\lesssim 36$ (DLS), and $\lesssim 75 \mathrm{yr}^{-1}$ per $10^{10} L_{B, \odot}$; the latter value is from Rau et al. (2008).

For the fiducial timescale of about one day (the upper bound of our survey), the volumetric rate limits are $\lesssim 0.3(-10 \mathrm{mag})$, $\lesssim 10^{-3}$ (-14 mag), and $\lesssim 4 \times 10^{-8}(-24 \mathrm{mag}) \mathrm{Mpc}^{-3} \mathrm{yr}^{-1}$. The rates at -14 and -24 mag are of interest since they are only an order of magnitude larger than the supernova and GRB rates, indicating that an expansion of our search might yield interesting limits on the rate of fast transients with $\sim 1$ day timescale.

\section{THEORETICAL EXPECTATIONS FOR THE LUMINOSITY OF FAST OPTICAL TRANSIENTS}

In the discussion above, we used fiducial peak absolute magnitudes of $-10,-14$, and -24 mag to infer limits on the volumetric rates of extragalactic FOTs. These particular values were chosen to match the most luminous classical novae, the least luminous supernovae, and typical long and short GRB afterglows, respectively. However, it is instructive to explore what peak luminosities are expected for sources with a fiducial timescale of $\sim 0.5 \mathrm{hr}$ to $\sim 1$ day for a range of potential mechanisms. We defer an exhaustive investigation of this question to future work and focus on the basic arguments here. At the most basic level, we note that explosive sources with a characteristic velocity of $\sim 10^{4} \mathrm{~km} \mathrm{~s}^{-1}$ that emit thermally with a peak in the optical $\left(T \sim 10^{4} \mathrm{~K}\right)$ will be limited to an absolute magnitude of $\gtrsim-14$; only sources undergoing relativistic expansion (e.g., GRBs) may significantly exceed this limits. We expand on this general theme below.

We first investigate the possibility of unusually fast and luminous classical novae. In general, novae follow the maximum magnitude versus rate of decline (MMRD) relation (e.g., della Valle \& Livio 1995), which indicates that faster novae are also more luminous. However, the MMRD relation flattens to a maximal observed value of about -10 mag for timescales shorter than a few days (della Valle \& Livio 1995). Indeed, the shortest observed timescales (quantified as $t_{2}$ or $t_{3}$, the timescales to decline by 2 or 3 mag, respectively) are $\approx 3-5$ days (Czekala et al. 2013). Theoretical models point to maximal magnitudes of about -10 and timescales as short as $t_{2} \approx 1$ day (Yaron et al. 2005). Moreover, the fraction of novae that achieve

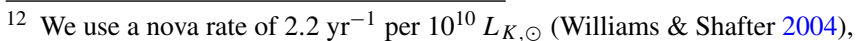
combined with the $K$-band luminosity density in the local universe of $4.4 \times 10^{8} L_{K, \odot} \mathrm{Mpc}^{-3}$ (Cole et al. 2001; Bell et al. 2003).
}

such high peak luminosity and rapid fading is $\lesssim 1 \%$ (e.g., Shafter et al. 2009), leading to a rate of $\lesssim 10^{-3} \mathrm{Mpc}^{-3} \mathrm{yr}^{-1}$, orders of magnitude below the upper bounds for -10 mag derived from the various searches (e.g., $0.3 \mathrm{Mpc}^{-3} \mathrm{yr}^{-1}$ for our search; Table 3). Thus, novae are not expected to contribute a population of luminous and fast extragalactic transients for current surveys.

We next explore the generic case of an explosive event with ejecta that cool adiabatically due to free expansion $\left(R=v_{\mathrm{ej}} t\right)$; i.e., a supernova-like explosion, but lacking internal heating. In this case, with the expansion driven by radiation pressure and using the standard diffusion approximation (Arnett 1982), the luminosity is given by

$$
L(t)=L_{0} \exp \left[-\frac{t}{\tau_{d, 0}}-\frac{t^{2}}{\sqrt{2} \tau_{d, 0} \tau_{h}}\right],
$$

where $\tau_{h}=R_{0} / v_{\mathrm{ej}}$ is the initial hydrodynamic timescale, $\tau_{d, 0}=B \kappa M_{\mathrm{ej}} / c R_{0}$ is the initial diffusion timescale, $\kappa$ is the opacity (for simplicity, we use $\kappa=\kappa_{\mathrm{es}}=0.4 \mathrm{~cm}^{2} \mathrm{~g}^{-1}$ ), $M_{\text {ej }}$ is the ejecta mass, $B \approx 0.07$ is a geometric factor, and $L_{0}=E_{0} / \tau_{d, 0}$ is the initial luminosity. A fiducial timescale of $0.5 \mathrm{hr}$ requires $R_{0} \lesssim 0.5 \mathrm{hr} \times v_{\mathrm{ej}} \approx 2 \times 10^{12}\left(v_{\mathrm{ej}} / 10^{4} \mathrm{~km} \mathrm{~s}^{-1}\right) \mathrm{cm}$; i.e., more extended sources are unlikely to produce transients with a duration as short as $\sim 0.5 \mathrm{hr}$. Using the appropriate size limit, we find that for a source with $L(0.5 \mathrm{hr}) / L_{0} \sim 0.1$ the resulting peak bolometric magnitude ranges from about $-8 \mathrm{mag}$ (for a white dwarf with $R_{0} \approx 10^{9} \mathrm{~cm}$ ) to about $-15 \mathrm{mag}$ (for a star with $\left.R_{0} \approx 10 R_{\odot}\right)$. The resulting characteristic effective temperature ranges from $T_{\text {eff }}=\left(L_{0} / 4 \pi R^{2} \sigma\right)^{1 / 4} \approx 2.2 \times 10^{4} \mathrm{~K}$ (for $R_{0} \approx 10^{9} \mathrm{~cm}$ ) to $\approx 1.1 \times 10^{5} \mathrm{~K}$ (for $R_{0} \approx 10 R_{\odot}$ ), or a peak in the ultraviolet. This means that the peak absolute magnitude in $g r$ will be a few magnitudes fainter, corresponding to the faint end of the magnitude range considered in the previous section (i.e., $\gtrsim-14$ mag).

The luminosity can be enhanced by appealing to an internal energy source, for example, radioactive heating as in the case of Type I supernovae. In this scenario, the luminosity is maximized if the radioactive decay timescale is well matched to the diffusion timescale, $\tau_{r} \approx \tau_{d} \approx B \kappa M_{\mathrm{ej}} / c R \approx 0.5 \mathrm{hr}$. The fast timescale investigated here requires different radioactive material than ${ }^{56} \mathrm{Ni}$, which powers the optical light curves of Type I supernovae, since the latter has $\tau_{r} \approx 8.8$ days. Instead, a better match may be provided by the radioactive decay of $r$-process elements, for which there is a broad range of timescales (e.g., Li \& Paczyński 1998; Metzger et al. 2010). Using a timescale of $0.5 \mathrm{hr}$, we find that the required ejecta mass is low, $M_{\mathrm{ej}} \approx 2 \times 10^{-6}\left(v_{\mathrm{ej}} / 10^{4} \mathrm{~km} \mathrm{~s}^{-1}\right)^{2} M_{\odot}$. For radioactive heating the total energy generation rate is $\epsilon(t)=\left(f M_{\mathrm{ej}} c^{2} / \tau_{r}\right) \mathrm{e}^{-t / \tau_{r}}$, where $f \ll 1$ is an efficiency factor, likely in the range of $10^{-6}-10^{-5}$ (Metzger et al. 2010). For the ejecta mass inferred above we find $\epsilon(t) \approx 10^{45} f \mathrm{erg} \mathrm{s}^{-1}$, and hence $L \sim 10^{40} \mathrm{erg} \mathrm{s}^{-1}$ (for $f \sim 10^{-5}$ ), or an absolute magnitude of about -12 mag. As in the discussion above, the spectrum will peak in the ultraviolet, and the optical emission ( $g$ band) will be dimmer by about 2 mag (i.e., to a peak of about $-10 \mathrm{mag}$ ). For a fiducial timescale of $\sim 1$ day, the peak absolute magnitude is larger, reaching $\approx-14 \mathrm{mag}$ in the optical. This is essentially the "kilonova" model invoked for compact object binary mergers ( $\mathrm{Li}$ \& Paczyński 1998; Metzger et al. 2010; Berger et al. 2013), with a predicted volumetric rate of $\sim 10^{-8}-10^{-5} \mathrm{Mpc}^{-3} \mathrm{yr}^{-1}$ (e.g., Abadie et al. 2010). We note, however, that recent opacity calculations for $r$-process material indicate that the actual peak brightness in the optical bands will be an order of magnitude 
fainter than suggested by the above calculation (Barnes \& Kasen 2013). A similar scenario is the thermonuclear Type Ia supernova model with heating by radioactive $\alpha$-chain elements $(\mathrm{Ca}$, Ti), leading to a peak optical brightness of $\sim-15 \mathrm{mag}$, but with a longer timescale of $\sim$ week even for low helium shell masses (Shen et al. 2010). Thus, models with radioactive heating are limited to $\sim-10 \mathrm{mag}(\sim 0.5 \mathrm{hr})$ or $\sim-14 \mathrm{mag}(\sim 1$ day $)$.

An alternative internal energy source is the spin-down of a newly born millisecond magnetar (Kasen \& Bildsten 2010; Woosley 2010). In this scenario, the maximal luminosity is achieved when the magnetar spin-down timescale, $\tau_{\mathrm{sd}} \approx 4 \times$ $10^{3}\left(B / 10^{14} \mathrm{G}\right)^{-2}(P / 1 \mathrm{~ms})^{2} \mathrm{~s}$, is comparable to the diffusion timescale, $\tau_{d} \approx 0.5 \mathrm{hr}$; here, $B$ is the magnetic field strength and $P$ is the initial rotation period. The available rotational energy is large, $E_{\mathrm{rot}} \approx 2 \times 10^{52}(P / 1 \mathrm{~ms})^{-2} \mathrm{erg}$, and therefore a magnetar engine can in principle produce extremely luminous fast transients, with a peak brightness of $L \approx E_{\mathrm{rot}} t_{\mathrm{sd}} / t_{d}^{2} \approx$ $5 \times 10^{48} \mathrm{erg} \mathrm{s}^{-1}$. However, since $\tau_{d} \approx 0.5 \mathrm{hr}$ requires a low ejecta mass of $\sim 10^{-6} M_{\odot}$, this scenario necessitates magnetar birth with negligible ejected mass, and results in a relativistic outflow; this is essentially the scenario invoked in magnetar models of GRBs. It is not obvious in this scenario whether the large energy reservoir will be emitted in the optical band, or primarily at $\mathrm{X}$-ray $/ \gamma$-ray energies. Thus, the optical signature may still be rather weak, and perhaps dominated instead by interaction with the ambient medium (i.e., an afterglow). Overall, the estimated magnetar birth rate is $\sim 10 \%$ of the core-collapse supernova rate (Kouveliotou et al. 1998), while the GRB rate is $\lesssim 1 \%$ of the supernova rate (Wanderman \& Piran 2010). This indicates that any magnetar-powered FOTs will have a rate orders of magnitude below the upper limits inferred by the existing searches.

Finally, luminous rapid optical transients can be produced by circumstellar interaction of a relativistic outflow, such as an onaxis ${ }^{13}$ GRB afterglow. The typical absolute magnitude of a shortduration GRB afterglow at $\delta t \approx 0.5 \mathrm{hr}$ is about -20 mag (based on a typical apparent brightness of about $22 \mathrm{mag}$ and a typical redshift of $z \approx 0.5$; Berger 2010). At this fiducial luminosity, the inferred volumetric upper limits from the FOT searches are $\lesssim 3 \times 10^{-5}$ (PS1/MDS), $\lesssim 3 \times 10^{-4}$ (DLS), $2 \times 10^{-3}$ (Fornax), and $\lesssim 7 \times 10^{-4}$ (ROTSE-III) $\mathrm{Mpc}^{-3} \mathrm{yr}^{-1}$. The actual volumetric rate of on-axis short GRBs is about $10^{-8} \mathrm{Mpc}^{-3} \mathrm{yr}^{-1}$ (Nakar et al. 2006), at least $3 \times 10^{3}$ times lower than the limits reached by the surveys. For long-duration GRBs the typical absolute magnitude at $\delta t \approx 0.5 \mathrm{hr}$ is much larger, about $-27 \mathrm{mag}$ (based on a typical apparent brightness of about 17 mag and a typical redshift of $z \approx 2$; Kann et al. 2010). At this luminosity, the volumetric upper limits for the various searches are ${ }^{14} \lesssim 10^{-6}$ (PS1/MDS), $\lesssim 2 \times 10^{-5}$ (DLS), $\lesssim 1 \times 10^{-5}$ (Fornax), and $\lesssim 3 \times 10^{-7}$ (ROTSE-III) $\mathrm{Mpc}^{-3} \mathrm{yr}^{-1}$. The inferred on-axis volumetric rate (at $z \sim 2$ ) is $\sim 10^{-8} \mathrm{Mpc}^{-3} \mathrm{yr}^{-1}$ (Wanderman $\&$ Piran 2010). Thus, unless there is a substantial population of relativistic explosions that do not produce $\gamma$-ray emission, the existing limits are much too shallow in the context of on-axis GRB rates.

We therefore conclude based on the comparison to various potential models and populations that

\footnotetext{
13 For significant off-axis angles the optical afterglow emission is expected to increase for days and then decline as a $\sim t^{-1}$ power law thereafter, leading to a characteristic timescale much longer than $0.5 \mathrm{hr}$.

14 We note that the volume accessible to our survey, as well as the DLS and the Fornax search is actually limited by their use of the $B$-band filter, which provides sensitivity only to $z \approx 4$ due to strong suppression by the Ly $\alpha$ forest.
}

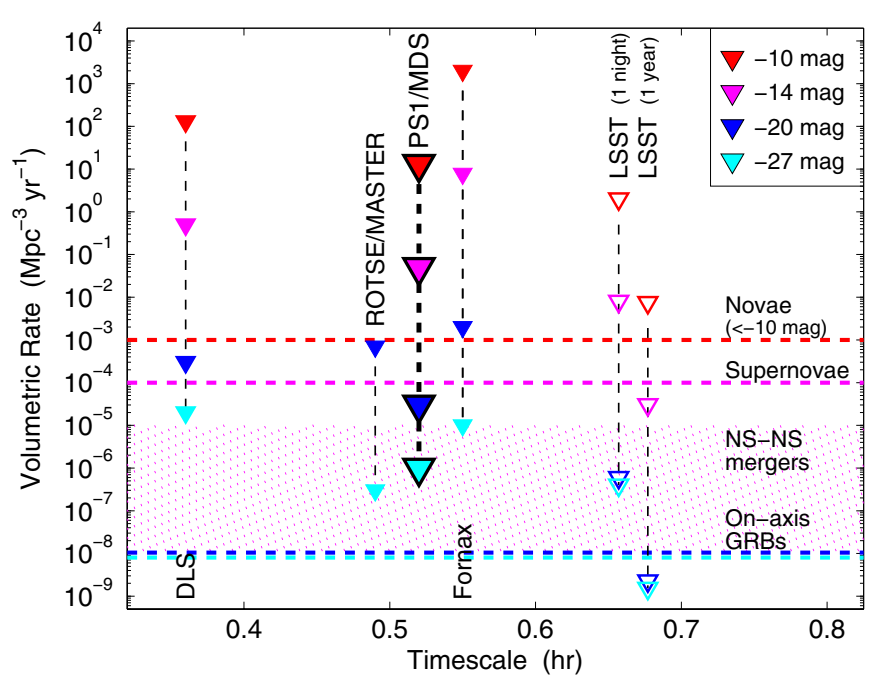

Figure 8. Limits on the volumetric rate of extragalactic fast optical transients as a function of timescale from our PS1/MDS survey and from the literature (Becker et al. 2004; Rykoff et al. 2005; Lipunov et al. 2007; Rau et al. 2008). For each survey we provide limits for a range of fiducial absolute magnitudes (top to bottom) of -10 (most luminous novae), -14 (least luminous $\mathrm{SNe}$ ), -20 (on-axis short GRB), and -27 (on-axis long GRB). Also marked are the actual volumetric rates of luminous novae, supernovae, binary neutron star mergers, and on-axis GRBs. The inferred limits from all existing surveys are orders of magnitude larger than the known volumetric rates, indicating that large new populations of currently unknown astrophysical fast transients are required for detectability, or that much larger surveys are essential to produce meaningful limits. The single night and full year limits for the LSST main survey indicate that this survey should detect some fast optical transients (the LSST estimates have been shifted along the time axis for clarity).

(A color version of this figure is available in the online journal.)

1. non-relativistic FOTs with a timescale of $\sim 0.5 \mathrm{hr}(\sim 1$ day) are generically limited to a peak optical brightness of $\sim-10(\sim-14)$ mag even if they are powered by radioactive heating; revised opacities for $r$-process matter may reduce these values by about an order of magnitude. Similarly, unusually fast classical novae are not likely to reach peak magnitudes larger than about -10 mag. Overall, the limits achieved by existing searches at these luminosities (Table 3) are orders of magnitude higher than the known and expected event rates for such transients (e.g., $\lesssim 10^{-3} \mathrm{Mpc}^{-3} \mathrm{yr}^{-1}$ for luminous novae); and

2. relativistic sources such as on-axis GRBs or transients powered by the spin-down of millisecond magnetars can produce much larger luminosities on a timescale of $\sim 0.5 \mathrm{hr}$, but the volumetric rate limits from the existing searches are still orders of magnitude larger than the very low known or anticipated rates of relativistic explosions. A possible exception is a fast transient recently discovered by Cenko et al. (2013), although with a timescale of $\sim f e w$ days.

In Figure 8, we summarize this information by comparing the volumetric rate limits from the various surveys at fiducial peak absolute magnitudes of $-10,-14,-20$ (short GRB), and -27 (long GRB) to the rates of known transients (e.g., novae, supernovae, neutron star binary mergers, on-axis short and long GRBs). The results demonstrate that the existing survey limits are orders of magnitude above the anticipated event rates based on the known classes. This indicates that much larger surveys are essential to robustly explore the transient sky on the $\sim$ hour-day timescale. In addition, we stress that given the low expected luminosity for non-relativistic fast transients $(\gtrsim-14)$, a profitable strategy is to systematically target nearby galaxies 
(as was done for example in the Fornax Cluster search; Rau et al. 2008) rather than image wide blank fields. On the other hand, searches for the rare but highly luminous relativistic fast transients are best focused on covering wide fields (preferably $\sim$ all-sky) at the expense of survey depth since the projected rate is at most $\sim$ few per sky per day, while the limiting factor for distance coverage is the use of optical filters (limited to $z \sim 6$ ) rather than depth.

\section{IMPLICATIONS FOR LSST}

Our search for FOTs proved highly effective at identifying foreground events without the need for expensive follow-up observations. This is unlike previous searches such as DLS and Fornax. This has been accomplished thanks to the use of dual-filter observations spanning a timescale of about $0.5 \mathrm{hr}$, with each observation composed of multiple exposures that help to eliminate individual spurious detections. In addition, the availability of color information ( $g-r$ in this case), coupled with the time baseline allowed us to effectively identify mainbelt asteroids near the stationary point. Similarly, deep multiband templates allowed us to unambiguously identify M-dwarf counterparts for all detected flares to a distance of at least $\sim 1.2 \mathrm{kpc}$ and with spectral types extending to about M9. With this information we were able to account for all the FOTs found in the survey, and to place the deepest limit to date on the rate of extragalactic fast transients. Our results are reassuring given that follow-up spectroscopy in the era of ongoing and future large surveys is in limited supply.

With this in mind, it is instructive to consider our results in the context of the anticipated LSST survey strategy (Ivezic et al. 2008). The LSST main survey is intended to cover a total of $\approx 18,000 \mathrm{deg}^{2}$, accounting for about $90 \%$ of the observing time. Each pointing will consist of two visits per field with a separation of about 15-60 minutes, with each visit consisting of a pair of $15 \mathrm{~s}$ exposures with a $3 \sigma$ depth of $\sim 24.5$ mag (or about $23.5 \mathrm{mag}$ at $10 \sigma$ ). It is anticipated that a total of about $3000 \mathrm{deg}^{2}$ will be imaged on any given night, potentially with more than one filter, leading to an areal exposure of about $62 \mathrm{deg}^{2}$ day in a single night, or about ${ }^{15} 1.6 \times 10^{4} \mathrm{deg}^{2}$ day $\mathrm{yr}^{-1}$, for the fiducial timescale of $0.5 \mathrm{hr}$. This survey will therefore achieve a sky projected rate limit (95\% confidence level) of $0.07 \mathrm{deg}^{-2} \mathrm{day}^{-1}$ in a single night and $3 \times 10^{-4} \mathrm{deg}^{-2} \mathrm{day}^{-1}$ in a year. The latter value represents a factor of 400 times improvement relative to our PS1/MDS search. In terms of a volumetric rate, the greater depth achieved by LSST will probe rates of $\approx 9 \times 10^{-3} \mathrm{Mpc}^{-3} \mathrm{yr}^{-1}$ for $-10 \mathrm{mag}$ and $\approx 3 \times 10^{-5} \mathrm{Mpc}^{-3} \mathrm{yr}^{-1}$ for $-14 \mathrm{mag}$ for $1 \mathrm{yr}$ of operations. At a typical brightness of short and long GRBs, the rate will be about $2 \times 10^{-9} \mathrm{Mpc}^{-3} \mathrm{yr}^{-1}$ (limited to $z \lesssim 6$ by the $z y$ filters), a few times deeper than the actual on-axis GRB rate, indicating that LSST is likely to detect such events.

The LSST data set can form the basis for a search similar to the one described here, with the main difference that contemporaneous color information may not be available. However, with an $\mathrm{S} / \mathrm{N} \approx 10$ cut as imposed here, the observations will reach about 1 mag deeper than our search, indicating that they will uncover M-dwarf flares to distances that are only about $50 \%$ larger than those found here (i.e., generally $\lesssim 2 \mathrm{kpc}$ ). At the same time, the LSST templates will reach a comparable depth to the PS1/MDS templates after only 10 visits per field per filter, i.e.,

\footnotetext{
15 Assuming $90 \%$ of the total observing time and a $20 \%$ loss due to weather
} and maintenance. within a year of when the survey commences. Thus, we conclude that it will be simple to identify M-dwarf counterparts in the template images for essentially all M-dwarf flares, negating the need for follow-up spectroscopy. This approach will effectively eliminate the largest known contaminating foreground for $\sim$ hour timescales.

Similarly, the time baseline of 15-60 minutes per field will allow for astrometric rejection of most asteroids even near the stationary point, although as we found in our PS1/MDS search, some asteroids have negligible motions that are indistinguishable from the astrometric scatter of field sources (2/8 in our search). Such asteroids can in principle be recognized using color information, but this is not likely to be available with LSST. On the other hand, a constant brightness level between visits 15-60 minutes apart, combined with a location near the ecliptic plane and in particular with solar elongation of $\sim 130^{\circ}$, will be indicative of an asteroid origin. Such fields can simply be avoided in searches for fast transients.

Thus, the two primary contaminants for extragalactic FOT searches (M-dwarf flares and asteroids near the stationary point) will be identifiable with the LSST baseline survey strategy. Furthermore, we anticipate based on the comparison to known transient classes that with a year of operations, LSST is unlikely to reveal large numbers of extragalactic fast transients unless they result from a source population that far exceeds classical novae, supernova progenitors, or compact object binaries (Figure 8).

\section{CONCLUSIONS}

We present a search for FOTs on a timescale of about $0.5 \mathrm{hr}$ to 1 day in consecutive $g_{\mathrm{P} 1} r_{\mathrm{P} 1}$ observations of the PS1/MDS fields, by requiring a detection in both filters with no additional detections on preceding or subsequent nights. The search yielded 19 astrophysical transients, of which eight events that lack quiescent counterparts are identified as mainbelt asteroids near the stationary point of their orbits, while the remaining 11 transients are identified as flares from M5-M9 dwarf stars at distances of about $0.2-1.2 \mathrm{kpc}$. The flare properties are generally similar to those from $\mathrm{M}$ dwarfs studied in the SDSS Stripe 82, although our sample extends to later spectral types and to much greater vertical distances from the Galactic plane.

A key result of our search is a limit on the sky-projected rate of extragalactic fast transients of $R_{\mathrm{FOT}} \lesssim 0.12 \mathrm{deg}^{-2} \mathrm{day}^{-1}$ ( $\sim 0.5 \mathrm{hr}$ ) that is about $30-50$ time deeper than previous limits; the limit for a timescale of $\sim 1$ day is $\lesssim 2.4 \times 10^{-3} \mathrm{deg}^{-2} \mathrm{day}^{-1}$. The upper bounds on the volumetric rates at fiducial absolute magnitudes of $-10,-14$, and -24 mag are likewise an order of magnitude deeper than from previous searches (Table 3). With an additional three years of PS1/MDS data in hand we can improve these estimates by a factor of a few. We also anticipate additional M-dwarf flare detections that will allow us to better characterize the distribution of flare properties, as well as the properties of flaring M dwarfs in general.

To guide the conversion from our sky-projected rate to volumetric rates, and to motivate future searches for fast transients, we also explore the expected luminosities of such transients for a range of physically motivated models. We find that non-relativistic fast transients are generally limited to about -10 mag for a timescale of $\sim 0.5 \mathrm{hr}$ and -14 mag for a timescale of $\sim 1$ day even if powered by radioactive decay. This is simply a reflection of the low ejecta mass required to achieve a rapid diffusion timescale of $\lesssim 1$ day. Such lowluminosity events are best explored through targeted searches 
of galaxies in the local universe, with anticipated event rates of $\lesssim 10^{-4} \mathrm{Mpc}^{-3} \mathrm{yr}^{-1}$ (Figure 8). A separate class of relativistic fast transients (on-axis GRBs, magnetar engines) can exceed $-25 \mathrm{mag}$ on a timescale of $\sim 0.5 \mathrm{hr}$, but they are exceedingly rare, with anticipated volumetric rates of $\sim 10^{-8} \mathrm{Mpc}^{-3} \mathrm{yr}^{-1}$ (or sky-projected rates of a few per sky per day). Such event rates are orders of magnitude below the level probed in current searches, but will be reached by the LSST main survey in a full year. Another strategy to find such events is a shallower search of a wider sky area than is planned for LSST. We note that a broad exploration of the various mechanisms that can power optical transients is essential to guide and motivate future searches for fast extragalactic transients. The initial investigation performed here already places clear bounds on the luminosities and rates for a wide range of mechanisms.

Finally, since the PS1/MDS survey is currently the only close analog to the main LSST survey in terms of depth, cadence, and choice of filters, we use the results of our search to investigate the efficacy of fast transient searches with LSST. We demonstrate that the main contaminants recognized here should be identifiable with the LSST survey strategy, without the need for expensive follow-up spectroscopy. Namely, asteroids near the stationary point can be recognized through a larger than average astrometric shift, a constant brightness in visits separated by 15-60 minutes, and an expected location near the ecliptic plane with solar elongation of $130^{\circ}$. LSST may not provide near-simultaneous color information, which can serve as an additional discriminant for asteroids. For M-dwarf flares we show that LSST's increased sensitivity will probe a larger volume of the Galaxy, but the correspondingly deeper templates will still allow for the identification of quiescent counterparts in essentially all cases. The PS1/MDS survey demonstrates that with multi-band photometry it is possible to identify M-dwarf flares without the need for follow-up spectroscopy (as in previous searches; e.g., Kulkarni \& Rau 2006). This result indicates that extragalactic FOTs should be able to pierce the veil of foreground flares.

We thank Mario Juric for helpful information on the planned LSST survey strategy. E.B. acknowledges support for this work from the National Science Foundation through grant AST-1008361. PS1 has been made possible through contributions of the Institute for Astronomy, the University of Hawaii, the Pan-STARRS1 Project Office, the Max-Planck Society and its participating institutes, the Max Planck Institute for Astronomy, Heidelberg, and the Max Planck Institute for Extraterrestrial Physics, Garching, The Johns Hopkins University, Durham University, the University of Edinburgh, Queen's University Belfast, the Harvard-Smithsonian Center for Astrophysics, and the Las Cumbres Observatory Global Telescope Network, Incorporated, the National Central University of Taiwan, and the National Aeronautics and Space Administration under grant NNX08AR22G issued through the Planetary Science Division of the NASA Science Mission Directorate.

Facility: PS1

\section{REFERENCES}

Abadie, J., Abbott, B. P., Abbott, R., et al. 2010, CQGra, 27, 173001

Arnett, W. D. 1982, ApJ, 253, 785

Barnes, J., \& Kasen, D. 2013, ApJ, 775, 18

Becker, A. C., Wittman, D. M., Boeshaar, P. C., et al. 2004, ApJ, 611, 418

Bell, E. F., McIntosh, D. H., Katz, N., \& Weinberg, M. D. 2003, ApJS, 149,289

Berger, E. 2010, ApJ, 722, 1946

Berger, E., Fong, W., \& Chornock, R. 2013, ApJL, 774, L23

Berger, E., Fong, W., Sanders, N., \& Chornock, R. 2012, ATel, 4619, 1

Bochanski, J. J., Hawley, S. L., \& West, A. A. 2011, AJ, 141, 98

Cenko, S. B., Kulkarni, S. R., Horesh, A., et al. 2013, ApJ, 769, 130

Cole, S., Norberg, P., Baugh, C. M., et al. 2001, MNRAS, 326, 255

Czekala, I., Berger, E., Chornock, R., et al. 2013, ApJ, 765, 57

della Valle, M., \& Livio, M. 1995, ApJ, 452, 704

Filippenko, A. V. 1997, ARA\&A, 35, 309

Gallagher, J. S., \& Starrfield, S. 1978, ARA\&A, 16, 171

Gorbovskoy, E. S., Lipunov, V. M., Kornilov, V. G., et al. 2013, ARep, 57, 233

Ivezić, Ž., Tabachnik, S., Rafikov, R., et al. 2001, AJ, 122, 2749

Ivezic, Z., Tyson, J. A., Acosta, E., et al. 2008, arXiv:0805.2366

Kaiser, N., Burgett, W., Chambers, K., et al. 2010, Proc. SPIE, 7733, 77330E

Kann, D. A., Klose, S., Zhang, B., et al. 2010, ApJ, 720, 1513

Kasen, D., \& Bildsten, L. 2010, ApJ, 717, 245

Kouveliotou, C., Dieters, S., Strohmayer, T., et al. 1998, Natur, 393, 235

Kowalski, A. F., Hawley, S. L., Hilton, E. J., et al. 2009, AJ, 138, 633

Kulkarni, S. R., \& Rau, A. 2006, ApJL, 644, L63

Leaman, J., Li, W., Chornock, R., \& Filippenko, A. V. 2011, MNRAS, 412, 1419

Li, L.-X., \& Paczyński, B. 1998, ApJL, 507, L59

Li, W., Chornock, R., Leaman, J., et al. 2011, MNRAS, 412, 1473

Lipunov, V. M., Kornilov, V. G., Krylov, A. V., et al. 2007, ARep, 51, 1004

Magnier, E. 2006, in The Advanced Maui Optical and Space Surveillance Technologies Conference, ed. S. Ryan (Red Hook, NY: Curran \& Associates, Inc.), E50

Mahabal, A., Drake, A., Djorgovski, S. G., et al. 2012, ATel, 4586, 1

Metzger, B. D., Martínez-Pinedo, G., Darbha, S., et al. 2010, MNRAS, 406, 2650

Nakar, E., Gal-Yam, A., \& Fox, D. B. 2006, ApJ, 650, 281

Rau, A., Ofek, E. O., Kulkarni, S. R., et al. 2008, ApJ, 682, 1205

Rest, A., Stubbs, C., Becker, A. C., et al. 2005, ApJ, 634, 1103

Rykoff, E. S., Aharonian, F., Akerlof, C. W., et al. 2005, ApJ, 631, 1032

Shafter, A. W., Rau, A., Quimby, R. M., et al. 2009, ApJ, 690, 1148

Shen, K. J., Kasen, D., Weinberg, N. N., Bildsten, L., \& Scannapieco, E. 2010, ApJ, 715, 767

Sokołowski, M., Małek, K., Piotrowski, L. W., \& Wrochna, G. 2010, AdAst, 463496

Tonry, J., \& Onaka, P. 2009, in Advanced Maui Optical and Space Surveillance Technologies Conference, ed. S. Ryan (Red Hook, NYC: Curran \& Associates, Inc.), E40

Tonry, J. L., Stubbs, C. W., Lykke, K. R., et al. 2012, ApJ, 750, 99

Wanderman, D., \& Piran, T. 2010, MNRAS, 406, 1944

Welsh, B. Y., Wheatley, J., Browne, S. E., et al. 2006, A\&A, 458, 921

Welsh, B. Y., Wheatley, J. M., Heafield, K., et al. 2005, AJ, 130, 825

West, A. A., Morgan, D. P., Bochanski, J. J., et al. 2011, AJ, 141, 97

Williams, S. J., \& Shafter, A. W. 2004, ApJ, 612, 867

Woosley, S. E. 2010, ApJL, 719, L204

Yaron, O., Prialnik, D., Shara, M. M., \& Kovetz, A. 2005, ApJ, 623, 398 